\title{
Hyperfine coupling and spin polarization in the bulk of the topological insulator $\mathrm{Bi}_{2} \mathrm{Se}_{3}$
}

\author{
S. Mukhopadhyay ${ }^{1}$, S. Krämer ${ }^{1}$, H. Mayaffre ${ }^{1}$, H.F. $\operatorname{Legg}^{1}$, M. Orlita ${ }^{1}$, \\ C. Berthier ${ }^{1}$, M. Horvatić ${ }^{1}$, G. Martinez ${ }^{1}$, M. Potemski ${ }^{1}$, B.A. Piot $^{1}$ \\ 1 Laboratoire National des Champs Magnétiques Intenses (LNCMI), \\ CNRS-UJF-UPS-INSA, F-38042 Grenoble, France \\ A. Materna ${ }^{2}$, G. Strzelecka ${ }^{2}$, A. Hruban ${ }^{2}$ \\ 2 Institute of electronic Materials Technology, ul. Wolczynska 133, 01-919 Warsaw, Poland
}

(Dated: June 20, 2018)

\begin{abstract}
Nuclear magnetic resonance (NMR) and transport measurements have been performed at high magnetic fields and low temperatures in a series of $n$-type $\mathrm{Bi}_{2} \mathrm{Se}_{3}$ crystals. In low density samples, a complete spin polarization of the electronic system is achieved, as observed from the saturation of the isotropic component of the ${ }^{209} \mathrm{Bi}$ NMR shift above a certain magnetic field. The corresponding spin splitting, defined in the phenomenological approach of a 3D electron gas with a large (spinorbit-induced) effective $g$-factor, scales as expected with the Fermi energy independently determined by simultaneous transport measurements. Both the effective electronic $g$-factor and the "contact" hyperfine coupling constant are precisely determined. The magnitude of this latter reveals a non negligible $s$-character of the electronic wave function at the bottom of the conduction band. Our results show that the bulk electronic spin polarization can be directly probed via NMR and pave the way for future NMR investigations of the electronic states in Bi-based topological insulators.
\end{abstract}

PACS numbers: 73.43.Qt, 73.40.Kp

Bismuth selenide, $\mathrm{Bi}_{2} \mathrm{Se}_{3}$, known for years as a narrow gap semiconductor, has recently appeared as one of the first examples of "3D topological insulators" [1-3]. As such unique state of matter, it is characterized by the coexistence of 2-dimensional conducting surface states with an insulating bulk material. The charge carriers at the surface behave as massless relativistic particles (Dirac fermions) with a spin locked to their translational momentum. These so-called "helical Dirac fermions", which promise applications in the field of spintronic [4] and quantum computation [5], have recently raised a considerable interest (see Ref. 6 for a review). As a matter of fact, the existence of gapless states at the boundary of the material is related to a well-defined change in the bulk band structure. In $\mathrm{Bi}_{2} \mathrm{Se}_{3}$, this originates from a parity inversion of the valence and conduction band in the presence of a large spin-orbit coupling [1].

In an effort to deepen our understanding of the spin properties of topological insulators, a characterization of the coupling between the charge carriers and the nuclei in the $\mathrm{Bi}_{2} \mathrm{Se}_{3}$ matrix is of high importance. Indeed, nuclear spins can inherently couple to the topologically protected electronic states and limit their coherence time. On the other hand, this hyperfine coupling can be efficiently exploited to probe the electronic system via NMR techniques. In particular, an electronic system bearing nonzero spin polarization acts as an effective local magnetic field which modifies the nuclei resonance frequency. This so-called "Knight shift" has previously been extensively studied to probe the electronic spin polarisation [7] as well as the spatial symmetry of the wave functions $[8,9]$ in some semiconductor-based bulk or low dimensional systems. A couple of recent works have investigated the
NMR properties of $\mathrm{Bi}_{2} \mathrm{Se}_{3}$ samples and revealed signatures of the bulk electronic states [10, 11]. These measurements were however limited to a narrow magnetic field and/or carrier density range, and did not systematically include a complete transport characterization of the bulk Fermi surface. The question of whether or not the electron spin degree of freedom can be reliably and directly accessed through such measurements is therefore still pending, and constitute the main motivation of this work.

In this work, we present a large body of NMR and transport measurements showing that the spin degree of freedom of bulk electronic states in $\mathrm{Bi}_{2} \mathrm{Se}_{3}$ can be probed via the hyperfine interaction with the $\mathrm{Bi}$ nuclei. This is evidenced by the observation of specific high magnetic fields features in the ${ }^{209} \mathrm{Bi} \mathrm{NMR}$ shift in $n$-type $\mathrm{Bi}_{2} \mathrm{Se}_{3}$ single crystals. In the lowest density samples, the complete spin polarization of the conduction electrons can be achieved, as indicated by the saturation of the isotropic component of the NMR shift above a density-dependent magnetic field. This allows us to precisely estimate the "contact term" of the hyperfine coupling, providing information on the spatial distribution of the electronic wave function, and to determine the amplitude and sign of an effective electronic $g$-factor describing the spin splitting in $\mathrm{Bi}_{2} \mathrm{Se}_{3}$. The "contact term" is of the same order of magnitude as in the typical semiconductors with $s$-like electron wave function in the conduction band, in spite of the $p$-type nature of electrons in $\mathrm{Bi}_{2} \mathrm{Se}_{3}$ anticipated in the theoretical models near the bulk band gap of this topological insulator $[1,12]$. A more complex magnetic field behavior is observed for the anisotropic component of the Knight shift usually related to $p$-like electrons. 
Our results pave the way for future unconventional experiments such as resistively $[13,14]$ or optically-detected NMR aiming at testing the spin-physics of the surfaces states in Bi-based topological insulators.

The $\mathrm{Bi}_{2} \mathrm{Se}_{3}$ crystals studied in this work were grown by the vertical Bridgman method [15]. Several batches with different amount of vacancies, known to act as a $n$-type dopant in $\mathrm{Bi}_{2} \mathrm{Se}_{3}$, were prepared to obtain $n$-type samples with different carrier concentration. A complete characterization of the samples has been obtained by using temperature- and angular-dependent magneto transport experiments [16] and shows that bulk transport is dominant in our systems. Our sample pool cover a Fermi energy range spanning $18-137 \mathrm{meV}$ from the bottom of the conduction band, corresponding to electron densities $n_{e}$ from $7.5 \times 10^{17} \mathrm{~cm}^{-3}$ to $1.6 \times 10^{19} \mathrm{~cm}^{-3}$. NMR measurements were performed both in superconducting magnets (6.5 - $17 \mathrm{~T}$ ) and in a $20 \mathrm{MW}$ resistive magnet (up to 30 T) [17].

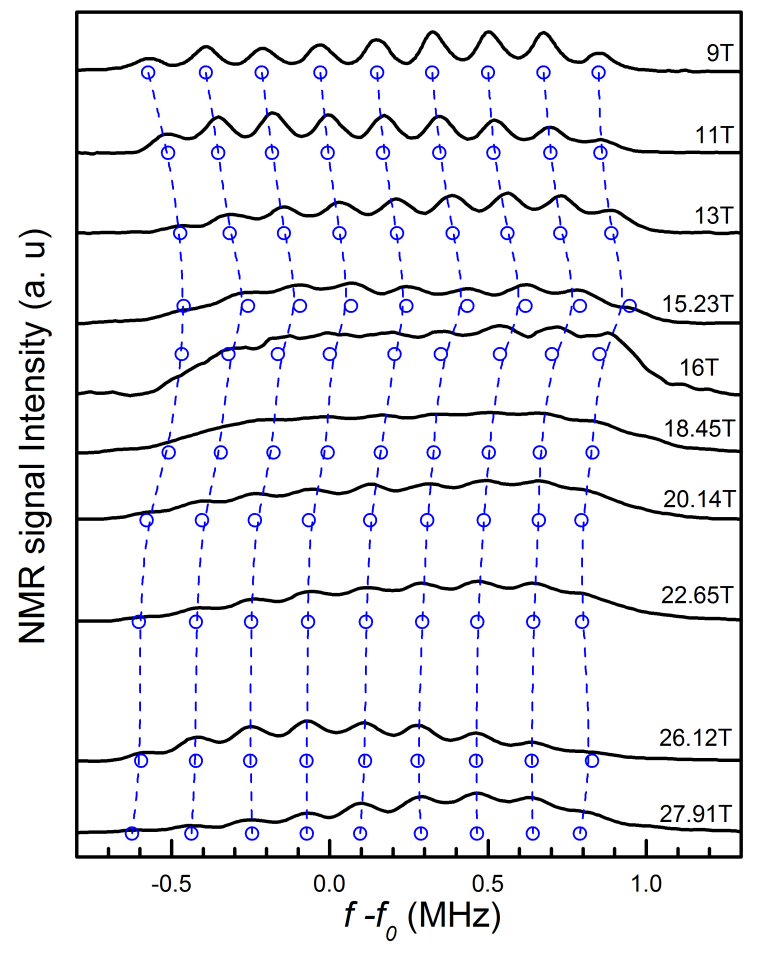

FIG. 1: (color online) Raw frequency-swept NMR spectra of sample P3-6 $\left(n_{e}=7.5 \times 10^{17} \mathrm{~cm}^{-3}\right)$ as a function of the magnetic field $B \| c$-axis at $T=5 \mathrm{~K}$. The vertical offset between the spectra is linear in $B$. The open circles denote the fitted NMR line positions, while the connecting dash lines are guide to eye. The frequency axis origin is defined by $\mathrm{f}_{0}={ }^{209}$ $\gamma B$, the resonance for the bare ${ }^{209} \mathrm{Bi}$ nuclei $\left({ }^{209} \gamma=6.84184\right.$ $\mathrm{MHz} / \mathrm{T}[18])$.

Figure 1 shows a typical quadrupolar split ${ }^{209} \mathrm{Bi}$ NMR spectrum at $5 \mathrm{~K}$ and its evolution with the magnetic field $B$ parallel to the crystal $c$-axis. The spectrum consists of a central line and four pairs of satellites due to the quadrupolar coupling between the nuclear spin $I=\frac{9}{2}$ charge distribution and the electric field gradients in the crystal. The main ${ }^{209} \mathrm{Bi}$ NMR central line is positively shifted compared to the expected position of the bare $\mathrm{Bi}$ resonance. In metals and semiconductor, this shift includes the "Knight shift", due to the interaction between the free carriers and the nuclei, which is our main focus of interest in this paper. As can be seen in figure 1, both the central line and its satellites shift to higher frequencies as the magnetic field increases up to $\sim 15 \mathrm{~T}$, where they show a sudden drop in frequency. The field dependence of the frequency shift $\left(f-f_{0}\right)$ of the ${ }^{209} \mathrm{Bi}$ NMR central line observed in figure 1 is plotted in figure 2 for three different samples.

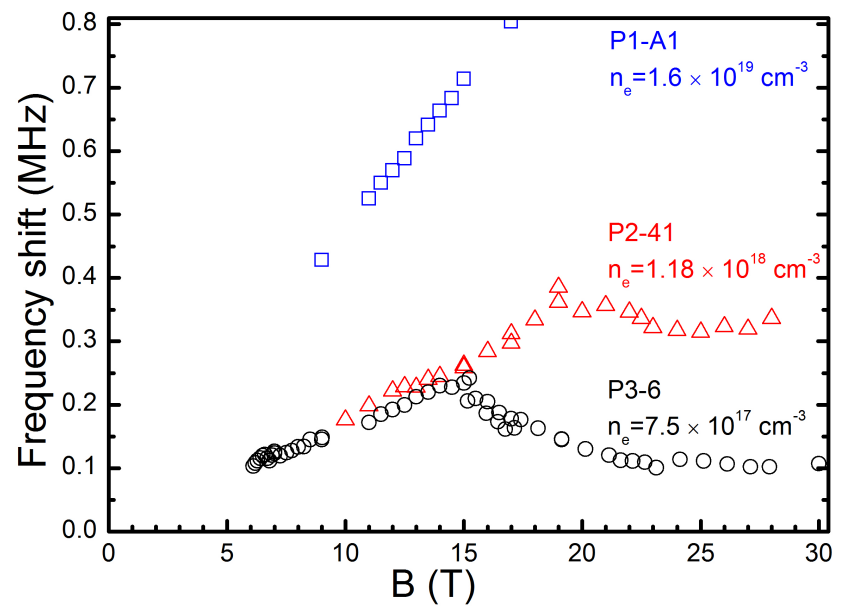

FIG. 2: (color online) NMR frequency shift $\left(f-f_{0}\right)$ of the $\mathrm{Bi}$ central line as a function of the magnetic field $B$ applied along the $c$-axis, for three samples having different carrier concentrations. $T=5 \mathrm{~K}$.

At low magnetic field, the shift increases (approximatively) linearly for all samples, with an average slope of about $0.0165 \mathrm{MHz} / \mathrm{T}(\sim 2410 \mathrm{ppm})$ for sample P3-6. At a fixed magnetic field, it increases with the electron density [19]. For samples P3-6 and P2-41 a pronounced kink appears in the NMR shift for magnetic fields of about 15 $\mathrm{T}$ and $21 \mathrm{~T}$, respectively. For the high density sample (P1-A1), no saturation is observed in the studied field range. These simple observations of density dependent features in the shift points towards the influence of the conduction electrons. In the simplest approach, the hyperfine contact term gives rise to an effective magnetic field proportional to the total number of free carriers $n_{e}$ and their spin polarization $P=\left(n_{\uparrow}-n_{\downarrow}\right) / n_{e}$, where $n_{\uparrow}$ and $n_{\downarrow}$ are the number of spin up and down in the system, respectively. This leads to an NMR frequency shift which is directly connected to the spin polarization of the system. In a bulk semiconductor the spin polarization is primarily determined by the ratio between the Zeeman 
and Fermi energies and increases with the magnetic field. In sufficiently high magnetic fields (or low Fermi energies), a complete spin polarization of the system can in principle be achieved, leading to a saturation of the internal effective magnetic field seen by the nucleus, and thus a saturation of the NMR frequency shift. This joint condition of a low Fermi energy and a high Zeeman energy can however only be experimentally realized in dilute 3D electronic systems under relatively high magnetic fields. To our knowledge, a saturation of the NMR Knight shift has only been observed in InSb so far [20-22], where the effective electron $g$-factor is about -51 .

In $\mathrm{Bi}_{2} \mathrm{Se}_{3}$, the bulk magneto-transport properties have been successfully described in the pioneering work of Köhler [23], using a model of a 3D electron gas with an energy-dependent anisotropic Fermi surface and a spin splitting described by an effective g-factor $g_{\text {eff }}^{*}=32 \pm 3$. This phenomenological approach was confirmed by our magneto-transport measurements [16]. A saturation of the $\mathrm{Bi}_{2} \mathrm{Se}_{3}$ Knight shift is thus expected for a magnetic field $B_{s}$, defined in the phenomenological approach by $g_{\text {eff }}^{*} \mu_{B} B_{s}=2^{\frac{2}{3}} E_{F}$, where $E_{F}$ is the zero-field Fermi energy [25]. For samples P3-6 and P2-41, our transport experiments yield $E_{F}=19.7$ and $26.4 \mathrm{meV}$ respectively, which with $g_{\text {eff }}^{*}=32.5$ gives corresponding values of $B_{s}$ $=16.6$ and $22.2 \mathrm{~T}$, close to the observed kinks in figure 2. The absence of any deviation of the shift in the higher carrier concentration sample is also expected since the much higher Fermi energy pushes $B_{s}$ far outside of our observable range. These very simple arguments points toward a direct connection between the electronic spin polarization and the NMR Knight shift in $\mathrm{Bi}_{2} \mathrm{Se}_{3}$.

We note, however, that for sample P3-6 a clear saturation of the Knight shift to a maximum value is not observed: the shift rather slowly drops down and appears to saturate to a lower value above $22 \mathrm{~T}$. This behavior has been confirmed for different samples of similar density and is also to some extent present for sample P2-41. For a deeper understanding of our data, one should consider the general expression of the hyperfine coupling Hamiltonian in metals or doped-semiconductors [26]:

$\mathcal{H}_{H F}=\frac{\mu_{0}}{4 \pi} g_{0} \mu_{B} \gamma \hbar \mathbf{I} \cdot\left[\frac{8 \pi}{3} \mathbf{S} \delta(\mathbf{r})-\left(\frac{\mathbf{S}}{r^{3}}-\frac{3(\mathbf{S} \cdot \mathbf{r}) \mathbf{r}}{r^{5}}\right)+\frac{\mathbf{l}}{r^{3}}\right]$,

where $g_{0}$ and $\mu_{B}$ are the electronic bare $g$-factor and Bohr magneton, respectively, $\gamma$ is the nuclear gyromagnetic ratio, $\mathbf{I}$ and $\mathbf{S}$ are the nuclear and electronic spin respectively, $\mathbf{r}$ is the electron-nuclei position vector and $\mathbf{l}$ the orbital momentum of the conduction electron. The first term in the bracket is the "contact" hyperfine interaction, which originates from the density probability of the electron exactly at the nuclear site $(r=0)$. This coupling is isotropic and dominates the hyperfine interaction in the case of an $s$-like electronic wave function. This yields a direct access to the electronic spin polarization (see e.g. Ref. 13, 14). The second term is anisotropic and results from the dipolar coupling between nuclear and electronic moment away from the $\mathbf{r}=0$ singularity, which dominates in the case of $p$-like electronic wave function (see e.g. Ref. 9, 27). The last term is related to the orbital momentum of the conduction electrons. In solids with non-degenerate orbital levels (which is the case for $\mathrm{Bi}_{2} \mathrm{Se}_{3}[1,12]$ ), the average orbital momentum $<\mathbf{l}>=0$, and this term usually only gives rise to a second order contribution [28]. However, in the presence of a strong spin-orbit coupling, a direct orbital contribution to the Knight shift may exist and be non-negligible: an example is the case of $p$-like electronic wave functions in $n$-type $\mathrm{PbTe}[8,29]$. As for all the terms in Eq.1, the relevance of this contribution is strongly dependent on the nature of the wave function, and no theoretical estimations of its strength in $\mathrm{Bi}_{2} \mathrm{Se}_{3}$ have so far been proposed [30]. Very recently, the presence of both anisotropic and isotropic contributions in the total NMR shift in $\mathrm{Bi}_{2} \mathrm{Se}_{3}$ has been reported at low carrier density [10]. To further investigate the origin of the observed downturn in high magnetic fields (Fig.2), we have performed angular dependent measurements of the NMR shift. For the axial symmetry, present in $\mathrm{Bi}_{2} \mathrm{Se}_{3}$, one can decompose the total frequency shift $\Delta f$ into two contributions, an isotropic contribution $\Delta f_{\text {iso }}$ and an anisotropic one $\Delta f_{\text {axial }}$, by writing $\Delta f=\Delta f_{i s o}+\Delta f_{\text {axial }}$. For a given angle $\theta$ between the magnetic field $B$ and the $c$-axis, the total shift can then be written with an explicit angular dependence as $\Delta f=\left[\mathcal{A}_{\text {iso }}+\left(3 \cos ^{2} \theta-1\right) \mathcal{A}_{\text {axial }}\right] n_{e} \times P(\theta)$. $\mathcal{A}_{\text {iso }}$ and $\mathcal{A}_{\text {axial }}$ are the isotropic and anisotropic hyperfine coupling constants, respectively, and the spin polarization $P$ may depend on $\theta$ in the case of anisotropic conduction band parameters. The isotropic and the axial part of the shift can be extracted from the rotational pattern of the central line [31]. Figure 3 shows the isotropic and axial components of the frequency shift for sample P3-6.

A clear saturation of the isotropic component of the Knight shift is observed above $16 \mathrm{~T}$, and can be attributed, as discussed above, to the full spin polarization of the electronic system manifesting itself directly through the "contact" term of the hyperfine coupling. The frequency shift can be calculated by writing $\Delta f_{i s o}=$ $\mathcal{A}_{\text {iso }} \times n_{e} \times P$, where the spin polarization $P$ is calculated in the simple 3D electron gas model introduced above. The two free parameters of this model, $g_{\text {eff }}^{*}$ and $\mathcal{A}_{\text {iso }}$, can be determined independently. As the Fermi energy is precisely estimated from transport measurements, $g_{\text {eff }}^{*}$ is only determined by the position of the saturating field $B_{s} . \mathcal{A}_{\text {iso }}$ is then adjusted to reproduce the frequency shift value, from the low field region to the saturation. The result of this simulation is plotted as a dotted line in figure 3 and catches the observed saturation very well [32]. An even better agreement with the experimental data can further be obtained by incorporating additional elements 


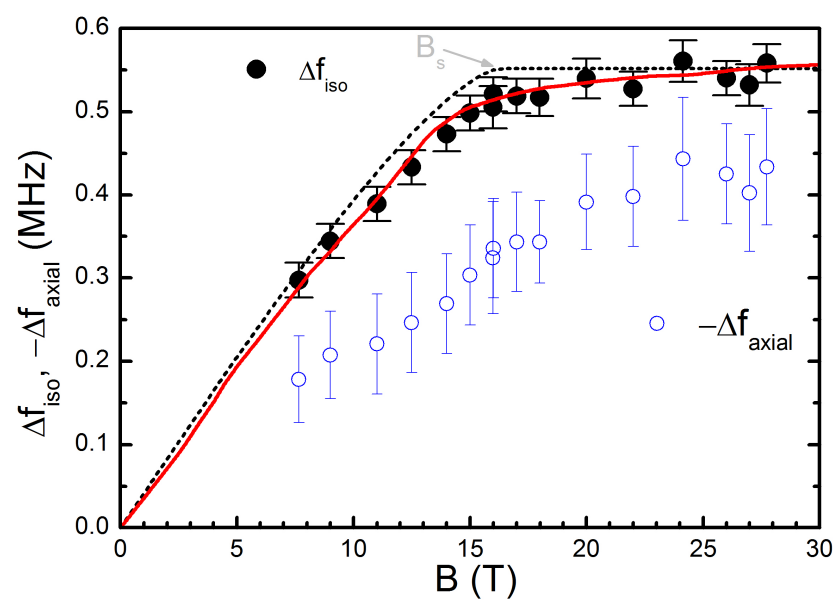

FIG. 3: (color online) Decomposition of the total frequency shift into isotropic $\left(\Delta f_{\text {iso }}\right)$ and axial parts $\left(\Delta f_{\text {axial }}\right)$ (see text) for sample P3-6. To compare the two components on the same graph, $-\Delta f_{\text {axial }}$ is plotted. The black dotted line is a simulations in a $3 \mathrm{D}$ electron gas model $\left(B_{s}\right.$ indicates the field corresponding to full spin polarization). The red solid line further includes effects of the Landau quantization and of disorder.

to our model, such as the Landau quantization of the electronic density of states (fully characterized from transport experiments [16]) and the effect of disorder which reduces the spin polarization through a level broadening. The result is plotted as solid line in figure 3 and gives a perfect quantitative description of our experimental data. Small modulations of the Knight shift can even be observed and attributed to the effect of the Landau quantization on the spin polarization, see e.g. the shallow downturn around $10.8 \mathrm{~T}$. In the case of $\mathrm{Bi}_{2} \mathrm{Se}_{3}$ the characteristics of these oscillations reflect, in particular, the peculiar commensurability of the spin and cyclotron gap [16], expected within the generalized 3D Dirac Hamiltonian for massive fermions [24]. The obtained effective electron $g$-factor is $g_{\text {eff }}^{*}=+32.8 \pm 1$, in a very good agreement with transport experiments performed on the same samples [16], and the previously reported value of $32 \pm 3$ [23]. We note that, in contrast to transport experiments, NMR experiments unambiguously determines the sign of the $g$-factor which directly impacts the sign of the Knight shift. The extracted value of the isotropic hyperfine coupling constant $\mathcal{A}_{\text {iso }}=6.94 \pm 0.16 \times 10^{-13} \mathrm{~cm}^{3} / \mathrm{s}$ enables us to estimate the density of the electronic wave function at the nuclear site $d_{\mathrm{Bi}}=8.72 \times 10^{25} \mathrm{~cm}^{-3}[33]$.

The obtained values of $\mathcal{A}_{\text {iso }}$ and $d_{\mathrm{Bi}}$ are comparable to or even higher than values determined for GaAs electron systems exhibiting pure $s$-like wave function (e.g. $\mathcal{A}_{\text {iso }}=$ $4.5 \pm 0.2 \times 10^{-13} \mathrm{~cm}^{3} / \mathrm{s}[34]$ and $d_{\mathrm{Ga}}=5.8 \times 10^{25} \mathrm{~cm}^{-3}[35]$ for ${ }^{71} \mathrm{Ga}$ in GaAs). This is at first sight surprising, since from a low energy expansion around the Dirac point [1], it is expected that the wave function describing the Dirac surface states, as well as the one describing the bulk conduction band minimum (CBM) and bulk valence band maximum (VBM) states, is of $p$-wave nature. In this case, the NMR shift produced by conduction electrons should be, at least at low energy, purely anisotropic. Our results, showing a very large isotropic component in the Knight shift for Fermi energies as low as $18 \mathrm{meV}$, therefore cannot be understood within this approach. They suggest that a non-negligible portions of the electronic wavefunction is of $s$-wave nature. In fact, our results could be consistent with very recent theoretical results [36] showing that about $10 \%$ of the electronic wave function at the $\mathrm{Bi}$ sites are of s-wave nature, emerging from deeper $\mathrm{Bi}$ " $6 s$ " orbitals taking part in shaping the wave function both in the VBM and CBM. Another interesting result of these calculations is the apparent absence of an $s$-like wave function at the Se site: this is consistent with the smaller shift observed at the same magnetic field for the ${ }^{77}$ Se NMR [37] and the extremely long relaxation time [37, 38], showing a weaker coupling of the Se nuclei to the conduction electrons. The remaining dominant $p$-wave nature of these wave functions leads to the anisotropic dipolar term in the ${ }^{209} \mathrm{Bi}$ NMR shift [39]. Its magnetic field behavior turns out to be more complex than the one of the isotropic component, as can be seen in figure 3. This illustrates the importance of having a sizeable isotropic component in the Knight shift as a reliable and direct access to the electronic spin polarization. We finally note that our temperature-dependent studies of the Knight shift [40], constitute an additional manifestation of the direct connection between the NMR shift and the electronic spin polarization and confirms our general approach.

In conclusion, combined high magnetic fields NMR and transport measurements have enabled us to evidence a direct link between the electron spin degree of freedom and the nuclear system, for the first time in a material of the topological insulator class. We precisely estimate the "contact term" of the hyperfine coupling and determine the amplitude and sign of an effective electronic $g$-factor phenomenologically describing the spin splitting in $\mathrm{Bi}_{2} \mathrm{Se}_{3}$. Our observation of a large isotropic NMR shift reveals a non-negligible proportion of $s$-like electronic states near the bulk band gap of this topological insulator.

This work was supported by the European Community under contract EC-EuroMagNetII-228043. M.O, M.P. and B.A.P acknowledge support from the ERC2012-AdG-320590 MOMB grant. Work in Poland has been supported by the research project: NCN UMO 2011/03/B/ST3/03362 Polska. 
[1] H. Zhang, C.-X. Liu, X.-L. Qi, X. Dai, Z. Fang, and S.-C. Zhang, Nat. Phys. 5, 438 (2009), ISSN 1745-2473.

[2] Y. Xia, D. Qian, D. Hsieh, L. Wray, A. Pal, H. Lin, A. Bansil, D. Grauer, Y. S. Hor, R. J. Cava, et al., Nat. Phys. 5, 398 (2009), ISSN 1745-2473.

[3] Y. L. Chen, J. G. Analytis, J.-H. Chu, Z. K. Liu, S.-K. Mo, X. L. Qi, H. J. Zhang, D. H. Lu, X. Dai, Z. Fang, et al., Science 325, 178 (2009).

[4] D. Pesin and A. H. MacDonald, Nat. Mater. 11, 409 (2012), ISSN 1476-1122.

[5] N. Read, Physics Today 65, 38 (2012).

[6] M. Z. Hasan and C. L. Kane, Rev. Mod. Phys. 82, 3045 (2010).

[7] S. E. Barrett, G. Dabbagh, L. N. Pfeiffer, K. W. West, and R. Tycko, Phys. Rev. Lett. 74, 5112 (1995).

[8] J. Y. Leloup, B. Sapoval, and G. Martinez, Phys. Rev. B 7, 5276 (1973).

[9] E. A. Chekhovich, M. M. Glazov, A. B. Krysa, M. Hopkinson, P. Senellart, A. Lemaitre, M. S. Skolnick, and A. I. Tartakovskii, Nat. Phys. 9, 74 (2013), ISSN 17452473.

[10] B.-L. Young, Z.-Y. Lai, Z. Xu, A. Yang, G. D. Gu, Z.-H. Pan, T. Valla, G. J. Shu, R. Sankar, and F. C. Chou, Phys. Rev. B 86, 075137 (2012).

[11] D. M. Nisson, A. P. Dioguardi, P. Klavins, C. H. Lin, K. Shirer, A. C. Shockley, J. Crocker, and N. J. Curro, Phys. Rev. B 87, 195202 (2013).

[12] Y. Cao, J. A. Waugh, X.-W. Zhang, J.-W. Luo, Q. Wang, T. J. Reber, S. K. Mo, Z. Xu, A. Yang, J. Schneeloch, et al., Nat. Phys. 9, 499 (2013), ISSN 1745-2473.

[13] L. Tiemann, G. Gamez, N. Kumada, and K. Muraki, Science 335, 828 (2012).

[14] M. Stern, B. A. Piot, Y. Vardi, V. Umansky, P. Plochocka, D. K. Maude, and I. Bar-Joseph, Phys. Rev. Lett. 108, 066810 (2012).

[15] A. Hruban, A. Materna, W. Dalecki, G. Strzelecka, M. Piersa, E. Jurkiewicz-Wegner, R. Diduszko, M. Romaniec, and W. Orlowski, Acta Physica Polonica A 120, 950 (2011).

[16] The transport experiments performed on our samples are presented in the supplemental material, section I.

[17] See supplemental material, section III.A for more experimental details.

[18] G. Carter, L. Bennett, and D. Kahan, Prog. Mater. Sci. 20, p.242 (1977), the value of ${ }^{209} \gamma$ in the International Union of Pure and Applied Chemistry (IUPAC) convention (R. K. Harris et al, Pure and Applied Chemistry 73, 1795-1818, (2009)), ${ }^{209} \gamma=6.841889$, is the same within 7 ppm.

[19] The density dependence of the total shift is analyzed in detail in the supplemental material section III.D., and shows in particular that the "chemical shift" is negligible in our system.

[20] G. A. Miranda, J. A. McNeil, and W. G. Clark, Phys. Rev. B 9, 495 (1974).

[21] A. Willig and B. Sapoval, Solid State Communications 11, 1077 (1972), ISSN 0038-1098.

[22] D. P. Tunstall, Journal of Physics C: Solid State Physics 21, 2853 (1988).

[23] E. W. H. Köhler, Phys. stat.sol. (b) 67, 675 (1975).

[24] M. Orlita et al., "Magneto-optics of massive Dirac fermions in bulk $\mathrm{Bi}_{2} \mathrm{Se}_{3}$ ", in preparation.

[25] This expression is obtained in the simple case of a 3D electron gas polarized under the effect of the Zeeman energy $g_{\text {eff }}^{*} \mu_{B} B$. This neglects the energy-dependent anisotropy of the Fermi surface, which is taken into account in the full calculation detailed in the supplemental material section II.A. and B.

[26] A. Abragam, Principle of Nuclear Magnetism, vol. p. 200 (Oxford University Press, 1985).

[27] J. Fischer, W. A. Coish, D. V. Bulaev, and D. Loss, Phys. Rev. B 78, 155329 (2008).

[28] J. Winter, Magnetic Resonance in Metals (Clarendon Press, 1971).

[29] R. L. Hota and G. S. Tripathi, Phys. Rev. B 44 1918 (1991), URL http://link.aps.org/doi/10.1103/ PhysRevB . 44.1918.

[30] We note that our study of the temperature dependence of the shift [40] suggests that, in our system, a spinindependent orbital contribution can be neglected in front of the spin-dependent ones.

[31] The complete procedure including the quadrupolar satellite fitting is detailed in the supplemental material, section III.B.2.

[32] The details of our simulations, which also includes the energy-dependent anisotropy of the effective mass, are given in the supplemental material in section II.A and B.

[33] The detailed calculation, as well as the possible role of core polarization and spin-orbit effects effects, are discussed in the supplemental material section III.B.3.

[34] N. N. Kuzma, P. Khandelwal, S. E. Barrett, L. N. Pfeiffer, and K. W. West, Science 281, 686 (1998).

[35] D. Paget, G. Lampel, B. Sapoval, and V. I. Safarov, Phys. Rev. B 15, 5780 (1977).

[36] A. Pertsova and C. M. Canali, arXiv:1311.0691v1 (2013).

[37] Results on the Se NMR are presented in the supplemental material, section III.C.

[38] R. E. Taylor, B. Leung, M. P. Lake, and L.-S. Bouchard, The Journal of Physical Chemistry C 116, 17300 (2012), http://dx.doi.org/10.1021/jp307051z.

[39] This is discussed in more detail in the supplemental material, section III.B.4.

[40] Results on the temperature dependence of the NMR signal are presented in the supplemental material, section III.E. 


\section{Hyperfine coupling and spin polarization in the bulk of topological insulator $\mathrm{Bi}_{2} \mathrm{Se}_{3}$ : SUPPLEMENTARY INFORMATION}

This supplemental material is organized in three main sections. Section I discusses the transport properties and conduction band parameters in $\mathrm{Bi}_{2} \mathrm{Se}_{3}$, section II formally presents the model used for our spin polarization and Knight shift simulations, and section III presents further details on our NMR experiments.

\section{TRANSPORT PROPERTIES AND CONDUCTION BAND PARAMETERS}

\section{A. Sample characteristics}

Magneto transport experiments were conducted on $\mathrm{Bi}_{2} \mathrm{Se}_{3}$ sample slices on which silver paste contacts were deposited in a "Hall bar-like" or Van der Pauw configuration. The measurements were performed using a standard low frequency lock-in technique in a variable temperature insert for temperature between 1.2 and $40 \mathrm{~K}$, and in magnetic fields up to $30 \mathrm{~T}$ produced by a $20 \mathrm{MW}$ resistive magnet. The characteristics of the studied sample are summarized in table $\mathrm{I}$, together with parameters extracted from the transport measurement analysis.

\begin{tabular}{|c|c|c|c|c|c|c|c|}
\hline & $\varepsilon(\mu \mathrm{m})$ & $\mathrm{T}_{D}(\mathrm{~K})$ & $F(\mathrm{~T})\left[F_{\|}(\mathrm{T})\right]$ & $m^{*}\left(m_{e}\right)$ & {$\left[\mathrm{m}_{\|}^{*}\left(m_{e}\right)\right]$} & $E_{F}(\mathrm{meV})$ & $n_{e}\left(\mathrm{~cm}^{-3}\right)$ \\
\hline P2-6A & 60 & $11.2^{a}$ & $19.6[28.8]$ & $0.123 \pm 0.005$ & {$[0.182 \pm 0.003]$} & 18.5 & $6.76 \times 10^{17}$ \\
\hline P2-6B & 60 & 9.6 & 21.2 & & - & 20.0 & $7.65 \times 10^{17}$ \\
\hline P3-6 & 120 & 9.6 & 20.9 & & - & 19.7 & $7.47 \times 10^{17}$ \\
\hline P2-41 & 10 & 15 & $28.0[42.6]$ & $0.123 \pm 0.007$ & {$[0.206 \pm 0.002]$} & 26.4 & $1.17 \times 10^{18}$ \\
\hline P1-3 & 10 & 17.4 & 154.8 & $0.130 ?$ & $7 \pm 0.002$ & 137 & $1.80 \times 10^{19}$ \\
\hline P1-A1 & 160 & - & 155.5 & & - & 137.9 & $1.81 \times 10^{19}$ \\
\hline
\end{tabular}

${ }^{a}$ for comparison, the corresponding $4 \mathrm{~K}$ mobility is $\mu=0.665 \mathrm{~m}^{2} / \mathrm{Vs}$

TABLE I: Characteristics and physical parameters of the $\mathrm{Bi}_{2} \mathrm{Se}_{3}$ samples: thickness $\varepsilon$, Dingle temperature $\mathrm{T}_{D}(\mathrm{~K})$, main frequency of the quantum oscillations $F(\mathrm{~T})$ (frequency $F_{\|}$for $B \perp c$-axis indicated in brackets when available), effective mass $m^{*}$ in units of the rest electron mass $m_{e}\left(\mathrm{~m}_{\|}^{*}\right.$ for $B \perp c$-axis indicated in brackets when available), Fermi energy $E_{F}$, and electron density $n_{e}$. The samples used for both transport and NMR measurements are highlighted in bold case.

The Shubnikov-De Haas oscillations ( $\mathrm{SdH}$ ) were observed in the low temperature magneto-transport and show, as usually observed, a single frequency connected to the single extremal cross section between the magnetic orbits and the Fermi surface. Representative results are shown for extremal values of our carrier density range in figure S1. The longitudinal resistance $R_{x x}$, symmetrized for positive and negative magnetic fields, shows quantum oscillations superimposed on a monotonously increasing background. The background can be removed either by subtracting a polynomial function to the data or by "adjacent averaging" method, and the corresponding oscillatory resistance is plotted in the upper left insets.

The sample quality was characterized by the determination of the Dingle temperature extracted from the field damping of the $\mathrm{SdH}$ oscillations. This was done using the standard Lifshitz-Kosevich formalism ${ }^{1}$ at the lowest temperatures. The resulting Dingle temperatures, reported in table I, reflect the typical trend of a disorder increasing with the carrier concentration, related to a higher concentration of Se vacancies in highly doped samples. The Hall voltage was also recorded to estimate the carrier density and, consistently with previous reports, ${ }^{2}$ an offset between the "Hall density" and the "SdH density" was observed with increasing carrier density. Because of geometrical errors possibly occurring in the determination of the electron density via the Hall voltage (particularly in anisotropic materials), quantum oscillations were considered to be a more reliable way of determining the carrier density (see also I.B.3. and III.D).

\section{B. Fermi surface anisotropy}

\section{Angular dependence of the quantum oscillations}

The angular dependence of the quantum oscillations was studied to map out the bulk conduction band Fermi surface for different values of the Fermi energy. SdH measurements were performed with an in-situ rotation stage, 

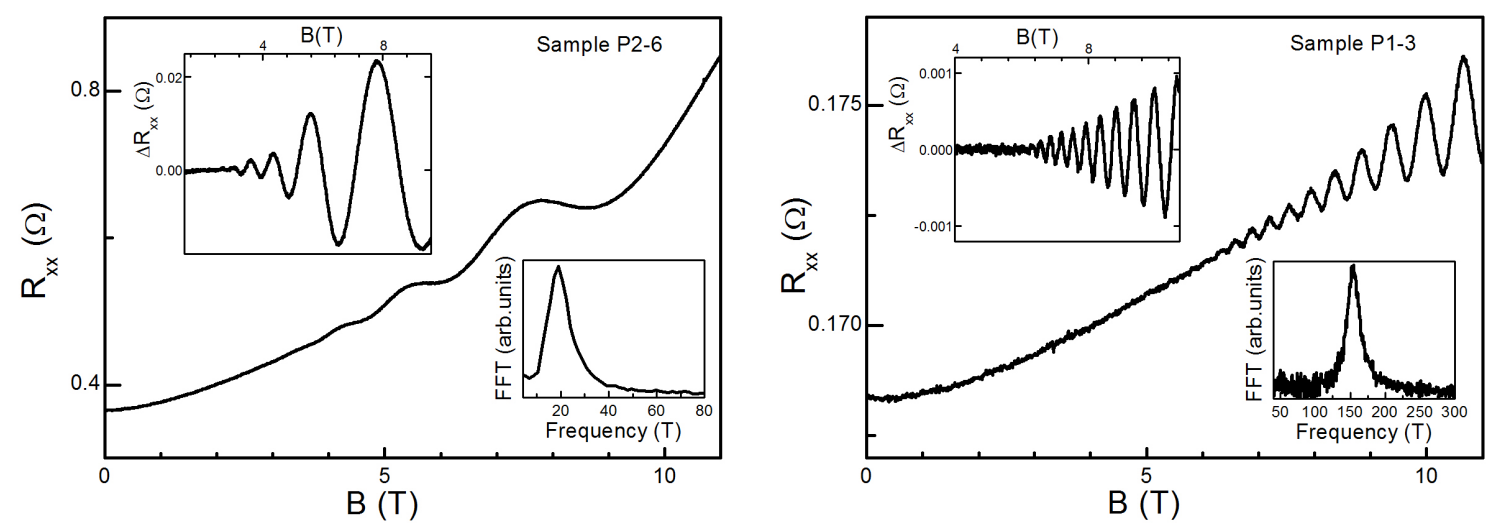

Figure S 1: Longitudinal resistance $R_{x x}$ versus total magnetic field $B \| c$-axis for sample P2-6 (left panel) and P1-3 (right panel) at $\mathrm{T}=1.4 \mathrm{~K}$. Upper left insets: oscillatory resistance $\Delta R_{x x}$. Lower right insets: Fourier transform of $\Delta R_{x x}(1 / B)$.

enabling us to tune the angle $\theta$ between the magnetic field and the crystal $c$-axis continuously from 0 to 90 degrees. The $\theta=0$ data were initially symmetrized by changing the polarity of the magnetic field to check that contact misalignment had a negligible impact on the Fourier transform analysis. As a double check, for a few samples the data were symmetrized for each angle and the Fourier transform analysis performed on the fully symmetrized data set, showing no noticeable difference compared to the analysis performed with non-symmetrized data. The variation of the oscillation frequency as a function of the tilt angle $\theta$ is reported in figure S2.a for three samples with different carrier concentration (increasing from P3-6 to P2-41 to P1-3, respectively).
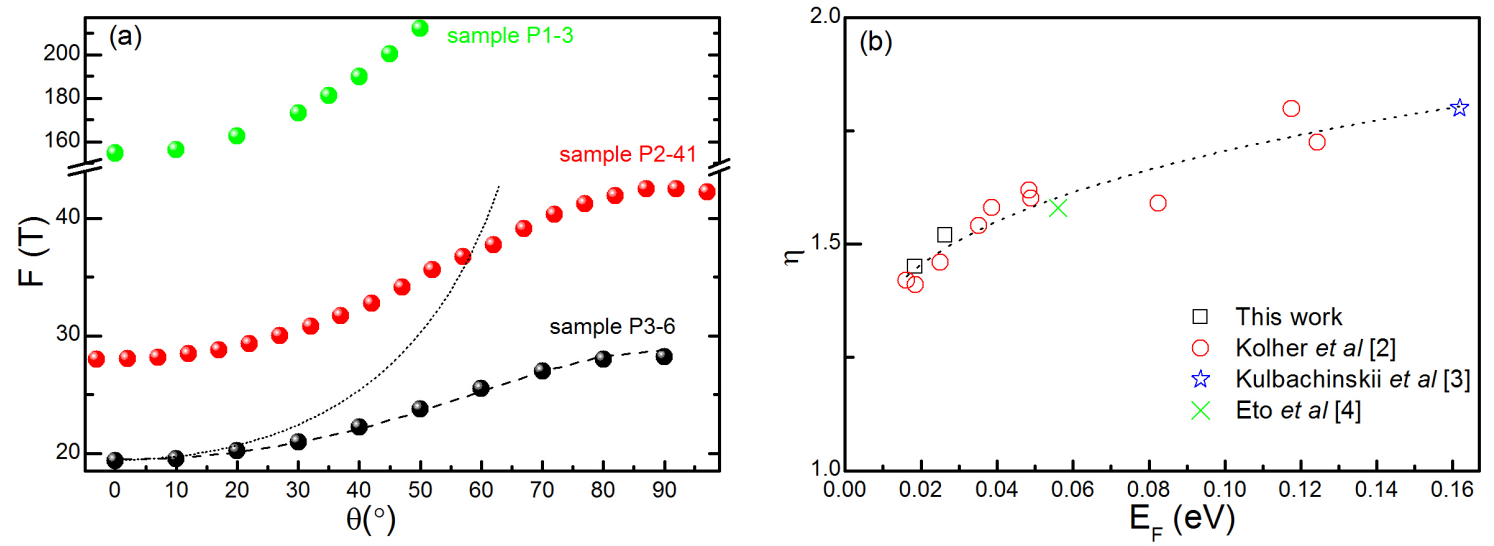

Figure S 2: (color online)(a) SdH oscillations frequency $\mathrm{F}$ as a function of the tilt angle $\theta$ for three samples with different carrier concentration (increasing from P3-6 to P2-41 to P1-3, respectively, from bottom to top). Calculated angular dependence of $\mathrm{F}$ for a 3D ellipsoidal Fermi surface (dashed-line), and for a $2 \mathrm{D}$ system (dotted-line). (b) Anisotropy $\eta=F_{\|} / F$ as a function of the Fermi energy in the conduction band, including previously published results. The dotted-line is a power-law fit of the data.

Since the oscillation frequency is directly proportional to the extremal cross section of the Fermi surface in the momentum space, the Fermi surface can be mapped out from this angular dependence. As can be seen in figure S2.a, the frequency $F$ increases as a function of $\theta$ in a way characteristic for an ellipsoidal Fermi surface (dashed-line), consistent with pioneering studies of the bulk conduction band at low energy. ${ }^{2}$ In this early work, the Fermi surface was described within a simple model of massive carriers with a parabolic (non-parabolic) dispersion in the $\mathrm{k}_{\perp}\left(\mathrm{k}_{\|}\right)$ direction, where $\mathrm{k}_{\perp}\left(\mathrm{k}_{\|}\right)$is the momentum in the direction perpendicular (parallel) to the $c$-axis of the crystal. This is associated with an increasing anisotropy of the Fermi surface observed as the Fermi level increases in the conduction band. Accordingly, in figure S2.a, the frequency increase with $\theta$ gets steeper as the carrier density increases (sample 
P3-6 to P2-41 to P1-3), confirming the higher anisotropy of the Fermi surface at higher Fermi energies. Another interesting aspect revealed by this data is that the cross section can not be perfectly reproduced by assuming a purely ellipsoidal Fermi surface above a certain Fermi energy. For example, fitting the low $\theta$ trend observed for sample P1-3 with an ellipsoidal model for the Fermi surface would give a much higher anisotropy than the one measured in such samples $^{2}$, suggesting that the Fermi surface is "squashed" along the $c$-axis. The ratio $F_{\|} / F$ of the $\mathrm{SdH}$ frequencies at $\theta=0^{\circ}$ and $\theta=90^{\circ}$, which reflect the anisotropy $\eta$ of the Fermi surface (independently of its shape), is plotted in figure S2.b together with results previously reported in the literature for samples with different carrier concentration. ${ }^{2-4}$ The determination of the Fermi energy is detailed below in section I.B.3. The dotted-line is a power-law fit of all the combined data sets used in our work to characterize the energy dependent anisotropy of the Fermi surface in $\mathrm{Bi}_{2} \mathrm{Se}_{3}$.

\section{Effective mass}

The temperature dependence of the SdH oscillations was measured for samples P2-6A, P2-41, P1-3 between 1 and $40 \mathrm{~K}$ for magnetic fields up to $11 \mathrm{~T}$. In figure S3, we report typical results obtained on sample P2-41, for temperatures between 1.2 and $30 \mathrm{~K}$.

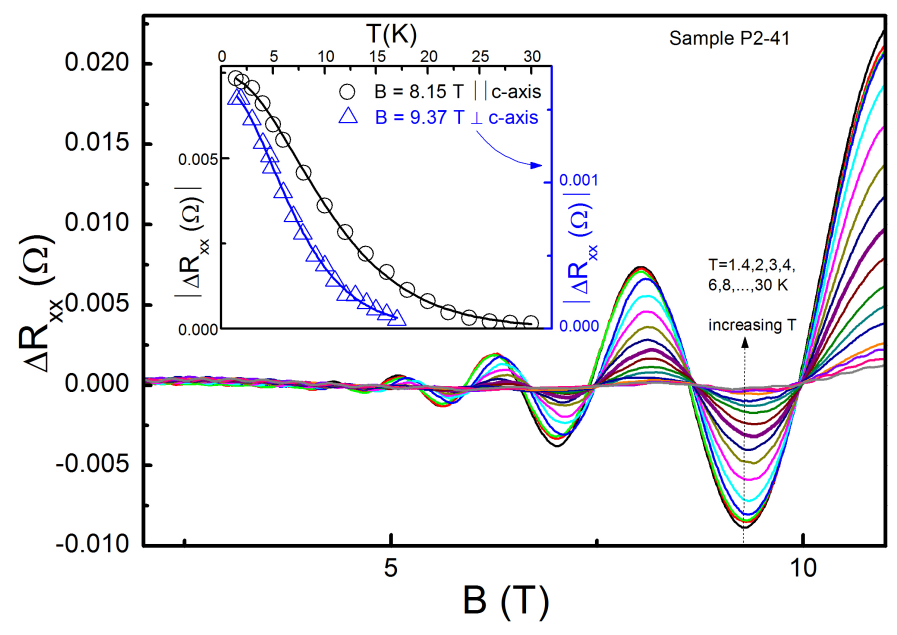

Figure S 3: (color online) Oscillatory magnetoresistance $\Delta R_{x x}$ versus total magnetic field for different temperatures for sample P2-41. Inset: temperature dependence of $\Delta R_{x x}$ at the resistance extremum at $\mathrm{B}=8.15 \mathrm{~T}$ for $B \| c$-axis (left-axis) and at $\mathrm{B}=9.37 \mathrm{~T}$ for $B \perp c$-axis (right-axis) (magneto resistance curve not shown).

In the inset of figure S3, we plot the temperature dependence of the oscillations amplitude at a fixed magnetic fields for different configurations: the standard $B \| c$-axis configuration (open circles), and the $B \perp c$-axis configuration (open triangles) where the magnetic field is in the $(a, b)$ plane of the crystal. The temperature damping can be very well-described by the Lifshitz-Kosevich formalism ${ }^{1}$, operational in this case of quantum oscillations of moderate amplitude. From this formalism one can extract an energy gap $\Delta$ separating the quantized energy levels, from which we can define an "apparent" effective mass for massive carriers, $m^{*}=(\hbar e B) / \Delta$. The values reported in table I are the one measured at the lowest possible magnetic fields, and were found to be almost constant over the field range studied. The effective mass in the $B \perp c$-axis configuration, $m_{\|}^{*}$, is also reported in table I. As can be seen, $m_{\|}^{*}$ is larger than $m^{*}$ and increases with the Fermi energy, while $m^{*}$ is approximatively energy independent in the studied range. This behavior is related to the energy-dependent anisotropy of the Fermi surface discussed in the previous section. In the case of a perfect ellipsoid, the ratio $m_{\|}^{*} / m^{*}$ is equal to the cross section ratio $F_{\|} / F$. The small differences between these two ratios for sample P2-41 shows the ellipsoidal model starts to breakdown already for Fermi energies above $25 \mathrm{meV}$.

\section{Fermi energy and carrier concentration}

From the frequency $F$ of the $\mathrm{SdH}$ oscillations, one can deduce the extremal Fermi surface cross section in the momentum space, $C S=\pi k_{F}^{2}$ (where $k_{F}$ is the Fermi wave vector), given by $C S=(\hbar F) /(2 \pi e)$. As argued above, 
an energy-independent effective mass is observed in the $B \| c$-axis configuration. One can therefore connect the Fermi energy $E_{F}$ to the extremal Fermi surface cross section $C S$ through the measured effective mass $m^{*}$, by writing $E_{F}=\left(\hbar^{2} C S\right) /\left(2 \pi m^{*}\right)$. This enables us to determine the Fermi energies reported in table I, which define the energy range probed in the conduction band in our experiment. The carrier density can then be calculated from the Fermi energy and the density of states (DOS) of the system. In such non-parabolic systems, the DOS has to be derived numerically from the actual dispersion relation. To simplify the problem, we approximate the DOS by introducing an energy-dependent effective mass along the $c$-axis, which aims at reproducing phenomenologically the energydependent anisotropy of the Fermi surface $\eta(E)$. The corresponding mass $m_{z}^{*}$ is given in an ellipsoidal Fermi surface approximation by $m_{z}^{*}=m^{*}(\eta(E))^{2}$, where $\eta(E)=1.32 E^{0.272}+1$ is determined from the fit of the data in figure S2 (dotted-line).

The spin degenerate density of states $D_{3 \mathrm{D}}(E)$ of the 3D Fermi sea is then approximated by:

$$
D_{3 \mathrm{D}}(E)=\frac{1}{2 \pi^{2}} \sqrt{\frac{2 m_{z}^{*}}{\hbar^{2}}} \frac{2 m^{*}}{\hbar^{2}} \sqrt{E}
$$

The carrier density $n_{e}$ is then:

$$
n_{e}=\int_{-\infty}^{E_{F}} D_{3 \mathrm{D}}(E) d E,
$$

The electron densities obtained are reported in table I. For the samples in which the effective mass was not directly measured, we have taken the effective mass value obtained for samples exhibiting a very similar oscillation frequency.

\section{High magnetic field transport and Zeeman energy}

The high magnetic field magneto transport was measured up to $30 \mathrm{~T}$ for samples P2-6B and P3-6, and is very similar to the data previously published in Ref. 5. In particular, the observed minima and maxima in the quantum oscillations above $B=15 \mathrm{~T}$ demonstrate that two Landau levels are below the Fermi energy at $B=15 \mathrm{~T}$. This, together with the absence of spin splitting in the quantum oscillation up to $30 \mathrm{~T}$, is fully consistent with the commensurability observed between the Zeeman and the cyclotron energies in $n$-type $\mathrm{Bi}_{2} \mathrm{Se}_{3} \cdot{ }^{3,5}$

By simulating the high field quantum oscillations with the same 3DEG model as the one used in Ref. 5, with the parameters $m^{*}$ and $T_{D}$ experimentally determined for our samples, one can draw the conclusion that the ratio $M$ between the Zeeman and cyclotron energies is:

$$
M=\frac{g_{e f f}^{*} \mu_{B} B}{\hbar \frac{e B}{m^{*}}}=2.0 \pm 0.2
$$

This gives an estimation of the electronic $g$-factor $g_{\text {eff }}^{*}=32.5 \pm 3$ for sample P2-6B and P3-6, in agreement with Ref. 5. We note that from the study of the phase of the quantum oscillations at high magnetic fields, the condition $M=1$ can be ruled out. The magnetic field dependence of the Fermi energy in the presence of a high Zeeman energy is also evidenced by a significant deviation of the phase of the quantum oscillations, as theoretically expected (see Ref. 6). This should be taken into account with great care when analyzing the phase of quantum oscillations to search for $2 D$ surface carriers in $\mathrm{Bi}_{2} \mathrm{Se}_{3}$ systems. Finally, the $\mathrm{SdH}$ phase analysis performed in the $B \perp c$-axis configuration up to $11 \mathrm{~T}$ enables us to evidence an anisotropy of the $g$-factor. For example, for sample P2-41 the $g$-factor in the crystal $(a, b)$ plane, $g_{\text {eff } \perp}^{*}$, is estimated to be $g_{\text {eff }}^{*} \perp=19 \pm 2$, whereas $g_{\text {eff }}^{*}=31 \pm 2$ along the $c$-axis. A similar anisotropy of the g-factor was also observed in recent spin resonance experiments at low magnetic fields ${ }^{7}$. This g-factor anisotropy, together with the previously discussed effective mass anisotropy, leads to the fulfillment of the coincidence condition $M \sim 2$ independently of the magnetic field orientation, in agreement with Ref. 5 . We finally note that the success of this phenomenological approach, in which the spin gap $g_{e f f}^{*} \mu_{B} B$ turns out to equal twice the value of the cyclotron gap associated with a parabolic in-plane dispersion, is not random but rooted to the $3 \mathrm{D}$ Dirac Hamiltonian for massive fermions applied to topological insulators with low electron-hole asymmetry and roughly parabolic bands. ${ }^{8}$ 


\section{KNIGHT SHIFT AND SPIN POLARISATION SIMULATIONS}

\section{A. Polarization of a 3D electron gas}

The simplest version of the model used to simulate the NMR Knight shift is based on the calculation of the electronic spin polarization of a 3D Fermi sea, characterized by an effective $g$-factor $g_{\text {eff }}^{*}$ defining the total spin splitting $g_{\text {eff }}^{*} \mu_{B} B$. One should note that the $g$-factor is different from the bare Zeeman $g$-factor since it includes the effect of the spin-orbit coupling to define an effective spin gap. As the magnetic field is turned on, the spin degenerate density of states of the 3D Fermi sea given by Eq. 1 is split by the Zeeman energy. The corresponding spin-up and spin-down populations are given by:

$$
n_{\uparrow, \downarrow}=\frac{1}{2} \int_{\mp\left(g_{\text {eff }}^{*} \mu_{B} B\right) / 2}^{E_{F}} D_{3 \mathrm{D}}\left(E \pm g_{\text {eff }}^{*} \mu_{B} B / 2\right) d E,
$$

and the Fermi energy is defined from the total electronic density $n_{e}$ :

$$
n_{e}=n_{\uparrow}\left(E_{F}, B\right)+n_{\downarrow}\left(E_{F}, B\right)
$$

This results in a field-dependant Fermi energy, which slowly decreases with the field until the point of full spin polarization is reached. At this point the Fermi energy follows the faster linear-in- $B$ decrease of the lower (fully occupied) spin branch of the density of states. This rapid motion of the Fermi energy with the magnetic field can be directly observed in the peak position of the SdH oscillations at high magnetic field (see also section I.C). The spin polarization under magnetic field is calculated using:

$$
P=\left(n_{\uparrow}-n_{\downarrow}\right) / n_{e}
$$

The frequency Knight shift $\Delta f_{\text {iso }}$ is then calculated as described in the main text by using $\Delta f_{\text {iso }}=\mathcal{A}_{\text {iso }} \times n_{e} \times P$, where $\mathcal{A}_{\text {iso }}$ is the (adjustable) hyperfine coupling constant.

\section{B. Landau quantization and disorder}

In bulk semiconductors or metal subjected to an intense magnetic field, the electron kinetic energy is modified by the Lorentz force, which leads to the formation of Landau bands originating from the intersection of the electron cyclotron orbits with the 3D Fermi surface. Assuming Lorentzian-shaped Landau levels (LL), the resulting "spin up" and "spin down" density of states can be written:

$$
D_{\mathrm{LL} \uparrow, \downarrow}=\frac{1}{2} \frac{\sqrt{m_{z}^{*}}}{2 \pi^{2} \hbar^{2}} e B \sum_{N_{\uparrow, \downarrow}=0}^{N_{\max }}\left[\frac{\left(E-E_{\mathrm{N} \uparrow, \downarrow}\right)+\sqrt{\left(E-E_{\mathrm{N} \uparrow, \downarrow}\right)^{2}+\Gamma^{2}}}{\left(E-E_{\mathrm{N} \uparrow, \downarrow}\right)^{2}+\Gamma^{2}}\right]^{0.5},
$$

where $E_{\mathrm{N}}$ are the LL eigen energy given by : $E_{\mathrm{N} \uparrow, \downarrow}=\left(N+\frac{1}{2}\right) \hbar \frac{e B}{m^{*}} \pm g_{e f f}^{*} \mu_{B} B$ for the "spin up" ("spin down") spin subbands. $\hbar \frac{e B}{m^{*}}$ is the cyclotron gap between Landau levels. $N$ is the Landau level index (a positive integer), and $N_{\max }$ is the Landau level index of the highest energy occupied LL. $\Gamma$ is the Landau level broadening related to the Dingle temperature $T_{D}$ by $\Gamma=\pi k_{B} T_{D}$, where $k_{B}$ is the Boltzmann constant. For each sample, $\Gamma$ is a therefore known and imposed from the $\mathrm{SdH}$ oscillation analysis. $m_{z}^{*}$ is still taking into account the energy dependant Fermi surface anisotropy as described above.

The Fermi energy is then obtained by solving the following equation :

$$
n_{e}=\int_{\left(\frac{1}{2}\right) \hbar \frac{e B}{m^{*}}+g_{e f f}^{*} \mu_{B} B-100 \Gamma}^{E_{F}}\left(D_{\mathrm{LL} \uparrow}(E)+D_{\mathrm{LL} \downarrow}(E)\right) d E,
$$

and the spin polarization is: 


$$
P=\frac{1}{n_{e}} \int_{\left(\frac{1}{2}\right) \hbar \frac{e B}{m^{*}}-g_{e f f}^{*} \mu_{B} B-100 \Gamma}^{E_{F}}\left(D_{\mathrm{LL} \uparrow}(E)-D_{\mathrm{LL} \downarrow}(E)\right) d E,
$$

The presence of the Landau bands creates an energy modulation of the 3D density of states which become important when the cyclotron gap exceeds the LL disorder broadening $\Gamma$. In the presence of a non-zero Zeeman energy, this can lead to an additional variation of the spin polarization in a magnetic field due to a different density of "spin down" and "spin up" states at the Fermi level, which superimposes on the usual "Zeeman-induced polarization". A particularity of $\mathrm{Bi}_{2} \mathrm{Se}_{3}$, which plays an important role in the determination of the behavior of the spin polarization, is the commensurability between spin and cyclotron gap $\left(\left(g_{e f f}^{*} \mu_{B} B\right) /\left(\hbar \frac{e B}{m^{*}}\right) \sim 2\right.$, see section I.C). This leads to a coincidence in the energy positions of the Landau levels $N_{\uparrow}$ and $(N+2)_{\downarrow}$, which tends to weaken the polarization oscillations because of the similarities of the "spin down" and "spin up" density of states at the Fermi level. However, in contrast with 2D systems where such perfect coincidence would lead to a quenching of the polarization oscillations in a magnetic field, in a 3D system some oscillations are still expected because the density of states at the Fermi level also involves the lowest Landau bands. These small oscillations are seen both in our calculations and experimental data in figure 3 in the main text. When the magnetic field reaches the value corresponding to the full spin polarization of a simple 3D Fermi sea, the two lowest Landau bands below the Fermi level are $(1)_{\downarrow}$ and $(0)_{\downarrow}$. Quantum oscillations can still be observed at higher magnetic fiels (as observed in transport), but no more variation of the polarization due to the Landau quantization is expected.

\section{Non-zero temperature}

The temperature dependencies are calculated by including the Fermi Dirac Distribution in the DOS integrations both for the simple 3D DOS as well as for the LL modified DOS. The Fermi level then shows the typical decrease at high temperature. If the temperature is large enough compared to the Fermi energy, this leads at fixed magnetic field to a drop of the polarization due to the depopulation of the lower spin band, which can be seen in the simulation in figure S7 (dotted-line) which will be discussed in the following section. If the sample Fermi energy is very large, no effect on the spin polarization can be seen and the Knight is relatively robust, as observed in the high density sample P1-A1. 


\section{NMR PROPERTIES}

\section{A. Methods}

The samples studied consist of thin flakes of $\mathrm{Bi}_{2} \mathrm{Se}_{3}$ crystal cleaved along the $(a, b)$ plane and their characteristics are gathered in table I. NMR measurements were initially conducted simultaneously with transport (i.e. within the same cooldown) on the central (contact-free) part of the sample to establish uniform experimental conditions. The observed good reproducibility of the sample properties from one cooldown to another has later on enabled us to perform NMR before the transport characterization to avoid potential perturbations due to the electrical contacts, and optimize the filling factor of the sample in the NMR coil. The magnetic field was calibrated by an ${ }^{27} \mathrm{Al}$ resonance of a $0.8 \mu \mathrm{m}$ thin aluminium foil placed inside the sample coil. Its surface area was slightly greater than the sample surface area (typically up to $3 \times 3 \mathrm{~mm}$ ) to estimate the field inhomogeneity over the sample, which was found to have a negligible effect on our results. In all our experiments, even in our thinnest samples, the NMR signal originates from the bulk of the sample due to the large ratio between the bulk and surface nuclei. This was confirmed by the absence of any thickness-dependant results in our study. The spectra were collected by summing equally spaced FFT spectra method ${ }^{9}$ using the standard Hahn echo sequence, $\pi / 2-\tau-\pi$. Because of the extremely thin coil thickness (150 - $30 \mu \mathrm{m}$, to ensure the optimum filling factor) the $\pi / 2$ pulse width could be kept very small, about $1 \mu s$. The very short spin-spin relaxation time, $T_{2}$, and the oscillation in the spin decay rate,${ }^{10}$ make the intensity distribution of the satellites and the central line anomalous. However, the anomaly is less severe if the spectra are recorded with a very short delay time between the pulses.

\section{B. Angular dependence and extraction of isotropic and anisotropic shift for ${ }^{209} \mathrm{Bi}$ NMR}

\section{Quadrupolar splitting}

${ }^{209} \mathrm{Bi}$ has a $\frac{9}{2}$ nuclear spin and thus shows four pairs of satellite resonance lines along with the central line, due to the quadrupolar coupling between the nuclei charge distribution and the electric field gradients (EFG) in the crystal. This can be seen in the NMR spectrum shown in figure 1 in the main text, where the quadrupolar satellites are separated by about $176 \mathrm{kHz}$. The position of the central line was determined by a multiple Lorentzian fit of the entire spectrum with a fixed quadrupolar splitting between the satellites. For P2-6, P3-6 and P2-41 samples the quadrupolar splitting, $\mathrm{f}_{Q}$, is $176 \pm 2 \mathrm{kHz}$, while for $\mathrm{P} 1$ it is $160 \pm 2 \mathrm{kHz}$, comparable to the previously reported values. ${ }^{10,11}$ We note that the second order quadrupolar shift, which can lead to a shift of the central line, is very small for such small $\mathrm{f}_{Q}$ (typically $\sim 1 \mathrm{kHz}$ at the lowest Larmor frequency) and is neglected here. For a quadrupolar nucleus in an electric field gradient, the satellite lines are generally progressively broader than the central line because the distribution of EFG due to defects leads to an additional "quadrupolar broadening". This expected hierarchy in the linewidth is not really observed in our samples, suggesting that the broadening is rather dominated by some other intrinsic factor. The observed global increase of the linewidth with the magnetic field supports a magnetic origin which remains to be clearly identified.

\section{Angular dependence of NMR}

The angular dependence of the ${ }^{209} \mathrm{Bi}$ NMR signal was studied in sample P3-6 for magnetic fields up to 30 T. The results obtained at $B=7.66 \mathrm{~T}$ are reported in figure $\mathrm{S} 4$. As the angle $\theta$ between the crystal $c$-axis and the magnetic field $B$ increases, the central line is right-shifted (larger total shift), and the total Knight shift reaches a maximum for $\theta=90^{\circ}$. The quadrupolar satellites follow a particular angular dependence in which all the lines collapse into the central line frequency for the "magic angle" $\theta=54.74^{\circ}$. To investigate this behavior more quantitatively, we note that $\mathrm{Bi}_{2} \mathrm{Se}_{3}$ has an axially symmetric EFG, with the principal axes of the EFG tensor coinciding with the ones of the hyperfine shift tensor. The angular dependence of the NMR line positions of a quadrupolar split spectra can then be written as: 

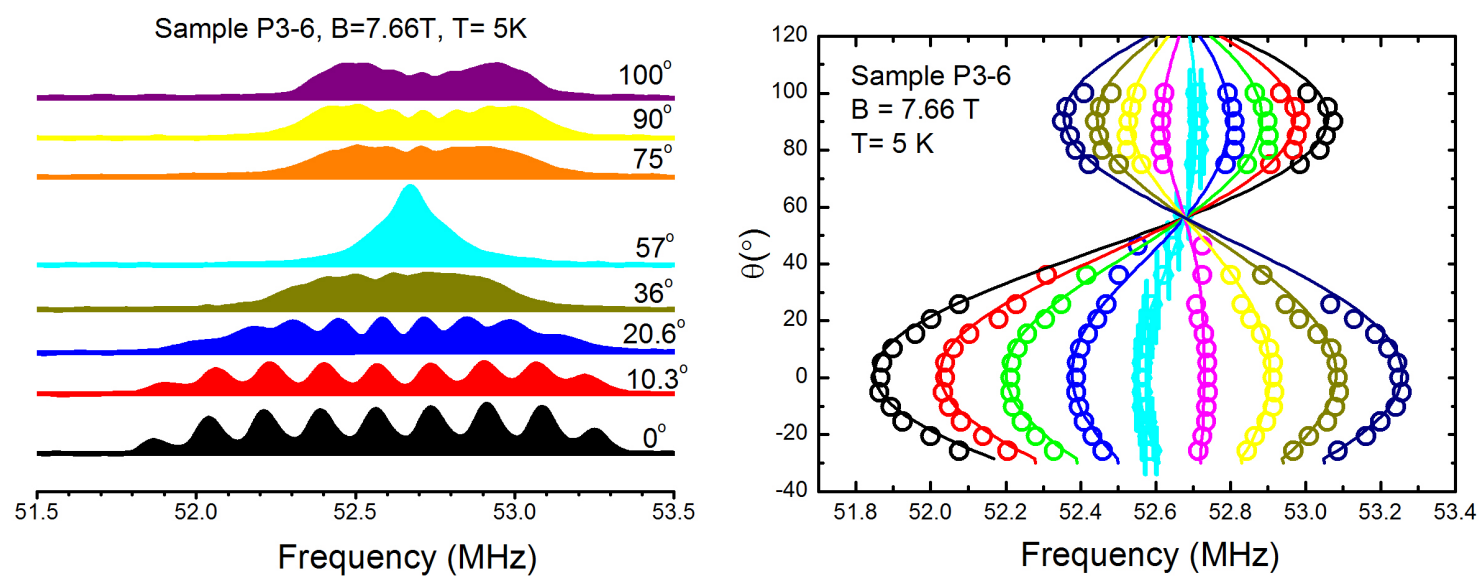

Figure S 4: Rotational pattern of the quadrupolar split ${ }^{209} \mathrm{Bi}$ spectra at $B=7.66 \mathrm{~T}$ and $T=5 \mathrm{~K}$. (a) Evolution of the spectra with the angle $\theta$. (b) Position of the resonances (open circles) obtained by fitting each spectrum with 9 Lorentzian functions, with proper constraints imposed by the spacing of the satellites. The solid lines are a fit of the angular dependence of the NMR spectrum with Eq. 11 (see text).

$$
\begin{aligned}
f(\theta)_{m \leftrightarrow(m-1)}= & { }^{209} \gamma B+\left[\mathcal{A}_{\text {iso }}+\left(3 \cos ^{2} \theta-1\right) \mathcal{A}_{\text {axial }}\right] n_{e} \times P(\theta) \\
& +\frac{f_{Q}}{2} \times\left(m-\frac{1}{2}\right)\left(3 \cos ^{2} \theta-1\right) \\
& +\frac{f_{Q}^{2}}{32 \gamma B}\left(1-\cos ^{2} \theta\right) \times\left\{[102 m(m-1)-18 I(I+1)+39] \cos ^{2} \theta-[6 m(m-1)-2 I(1+1)+3]\right\}
\end{aligned}
$$

where $\mathcal{A}_{\text {iso }}$ and $\mathcal{A}_{\text {axial }}$ are the isotropic and anisotropic hyperfine coupling constants, respectively, $P(\theta)$ the electronic spin polarization, ${ }^{209} \gamma$ is the ${ }^{209} \mathrm{Bi}$ gyromagnetic ratio, $I$ the nuclear spin, $m$ the magnetic quantum number for $I$, and $f_{Q}$ is the nuclear quadrupole frequency. The first term (first line, omitting the reference frequency ${ }^{209} \gamma B$ ) is the same as the one appearing in the main text and describes the frequency shift of the central line. The axial term is weighed by the factor $\left(3 \cos ^{2} \theta-1\right)$ which originates from the integration on the dipolar term in Eq. 1 in the main text. The second term (second line) describes the angular evolution of the quadrupolar splitting satellites. The last term in $\{. .$.$\} (third line) is the second order quadrupolar shift of the lines, which for our lowest Larmor frequency$ is only $\sim 1 \mathrm{kHz}$ and $\sim 6 \mathrm{kHz}$ for the central line and fourth satellite transitions, respectively, and can therefore be neglected. Eq. 10 can be re-written as:

$$
\begin{aligned}
f(\theta)_{m \leftrightarrow(m-1)}= & { }^{209} \gamma B+\left[\Delta f_{\mathrm{i} s o}+\frac{\Delta f_{\text {axial }}}{2} \times\left(3 \cos ^{2} \theta-1\right)\right] \times \frac{P(\theta)}{P(0)} \\
& +\frac{f_{Q}}{2} \times\left(m-\frac{1}{2}\right)\left(3 \cos ^{2} \theta-1\right),
\end{aligned}
$$

where $\Delta f_{\text {iso }}=\mathcal{A}_{\text {iso }} n_{e} \times P(0)$ and $\Delta f_{\text {axial }}=2 \mathcal{A}_{\text {axial }} n_{e} \times P(0)$ are the isotropic and axial component of the frequency shift at $\theta=0^{\circ}$, respectively. The total frequency shift of central line at $\theta=0^{\circ}$ is $\Delta f=\Delta f_{\text {iso }}+\Delta f_{\text {axial }}$. Because of the anisotropy of the conduction band $g$-factor (see I.C), the polarization appearing in Eq. 10 and 11 may also be, depending on the value of the magnetic field, anisotropic. This leads to a $\theta$-dependant pre-factor which applies both to the isotropic and axial component of the shift. We note that observing a central line which is independent of $\theta$ in this system therefore does not mean that the axial component is absent, but simply that the $\theta$-dependence due to the axial term is compensated by the anisotropic polarization. We have taken this effect into account by including the $P(\theta)$ dependence calculated in our model (see II. A) with $g_{\text {eff }}^{*} \perp=23$, which we obtained by using the $g$-factor anisotropy ratio observed in our low density samples (see I.C), and $g_{\text {eff }}^{*}=32.8$.

The fits of our experimental data using this equation, shown as solid lines in figure S4, are very good. This enables us to determine the two fitting parameters, $\Delta f_{\text {iso }}$ and $\Delta f_{\text {axial }}$, for a given magnetic field. In principal, these parameters are uniquely defined by fitting the central line, since for $m=1 / 2$, the right side of Eq. 11 is reduced to the first term. Fitting the satellites (whose angular dependence with respect to the main line is known) further confirms the 
displacement of the central line and enhances the fit accuracy. This process is repeated over our total magnetic field range (6-30 T) to obtain the magnetic field dependence of the isotropic and anisotropic components of the Knight shift, which are reported in figure 3 in the main text. In high magnetic fields $(>15 \mathrm{~T})$ the rotational pattern was only measured for 5-10 angles around $\theta=0^{\circ}$, and at $\theta=54.74^{\circ}$ where all the satellites collapse on to the central line, giving a better precision for the fit. While the spectra were collected by frequency sweeps in the superconducting magnet $(6.1-17 \mathrm{~T})$, most of the high field spectra $(15-30 \mathrm{~T})$ were collected by field sweep in the resistive magnet. For a field sweep spectrum, an equation equivalent to Eq. 11 in the field domain was used to find the isotropic and axial part of the shift.

\section{Isotropic shift}

As can be seen in figure 3 in the main text, the isotropic component shows a clear saturation which can be perfectly reproduced by our model. This enables us to extract the isotropic hyperfine coupling constant $\mathcal{A}_{\text {iso }}$, and the density of the electronic wave function at the nuclear site. We note that, as mentioned before ${ }^{12-14}$, the determination of $\mathcal{A}_{\text {iso }}$ in the quantum limit is highly reliable because the spin polarization is full and independent of the band structure parameters. Our model further shows that if the band parameters are well mastered (e.g. from transport experiments), a single value of $\mathcal{A}_{\text {iso }}$ can be used on the whole magnetic field range. Using our simple 3DEG model (dotted line in figure 3 in the main text), one obtains $\mathcal{A}_{\text {iso }}=7.41 \times 10^{-13} \mathrm{~cm}^{3} / \mathrm{s}$, whereas the more complete model including Landau quantization and disorder (solid red line in figure 3 in the main text) gives $\mathcal{A}_{\text {iso }}=6.94 \pm 0.16 \times 10^{-13} \mathrm{~cm}^{3} / \mathrm{s}$, which is the value we retain. The corresponding value $d_{\mathrm{Bi}}$ of the density of the electronic wave function at the nuclear site, is obtained as followed. From Eq. 1 in the main text, the "contact" term of the hyperfine coupling Hamiltonian is:

$$
\mathcal{H}_{\text {contact }}=\frac{\mu_{0}}{4 \pi} g_{0} \mu_{B} \gamma \hbar \mathbf{I} \cdot \frac{8 \pi}{3} \mathbf{S} \delta(\mathbf{r})=\frac{2}{3} \mu_{0} g_{0} \mu_{B} \gamma \hbar \mathbf{I} \cdot \mathbf{S} \delta(\mathbf{r})
$$

For a nucleus $i$ at a position $r_{i}$, this Hamiltonian is equivalent to a Zeeman interaction in a magnetic field defined by:

$$
\mathbf{B e}=-\frac{2}{3} \mu_{0} g_{0} \mu_{B} \sum_{q} \mathbf{S}_{q}\left|\psi_{q}\left(r_{i}\right)\right|^{2}
$$

where the summation is done on the occupied electronic states of wave function $\psi_{q}$. When the spin polarization of the $n_{e}$ conduction electron is full, the corresponding frequency shift is then:

$$
\Delta f_{\text {iso }}=\frac{2}{3} \mu_{0} g_{0} \mu_{B} \gamma\left(d_{\mathrm{Bi}} \Omega\right) \frac{1}{2} n_{e}
$$

where $d_{\mathrm{Bi}}$ is the density of the electronic wave function at the nuclear site normalized on the primitive cell volume $\Omega$. The rhombohedral primitive cell in $\mathrm{Bi}_{2} \mathrm{Se}_{3}$ has a volume $\Omega=1.497 \times 10^{-23} \mathrm{~cm}^{3}$. The factor $\frac{1}{2}$ originates from the projected value $S_{z}=\frac{1}{2}$ of the spin number in the fully polarized regime. This equation and the definition of $\Delta f_{i s o}$ in III.B.2 defines the link between $\mathcal{A}_{\text {iso }}$ and $d_{\mathrm{Bi}}$, and we can deduce the value of $d_{\mathrm{Bi}}=8.72 \times 10^{25} \mathrm{~cm}^{-3}$.

We should point out that the extracted value of $\mathcal{A}_{\text {iso }}$ and $d_{\mathrm{Bi}}$ are relevant for a Fermi energy $E_{F}=19.7 \mathrm{meV}$ (sample P3-6), and may show a dependence on the position of the Fermi level in the conduction band. For the sake of simplicity, we have also omitted the relativistic corrections which are known to enhance the value of $\mathcal{A}_{\text {iso }}$ by a factor of the order of $3 .^{15}$ Additionally, the extracted value includes possible "core polarization" effects. The core polarization originates from an asymmetry of the spin part of the inner core $s$-shell wave function which develops due to the proximity of unpaired electrons in the conduction band. For heavy elements, this non-zero polarization can lead to a modification of the "contact" hyperfine coupling. In $\mathrm{Bi}_{2} \mathrm{Se}_{3}$ the unpaired electrons are in the $6 p$ shell with a possible contribution from $6 s$ electrons (this is discussed in the main text). The core polarization shift arising from $6 p$ electrons is expected to be negative. ${ }^{16}$ However, contributions from $s$-shells are always positive, and generally considered as an amplification of the usual contact term. The large positive isotropic shifts we observed in our work suggest that core polarization effects, if important, would rather arise from $s$ electrons. A better understanding of these effects would require new theoretical studies and is out of the scope of the present experimental paper. Finally, we would like to discuss the possible role of spin-orbit effects in the isotropic shift. It was theoretically proposed that spin-orbit effects could lead to an additional contribution to the Knight shift, ${ }^{17}$ which in some systems would manifest itself as an enhancement of the isotropic shift. A signature of this contribution is predicted to be visible in the low temperature dependence of the Knight shift. In our samples, this signature is absent, as shown in our study of the 
temperature dependence of the Knight shift (see III.E), suggesting that this spin-orbit contribution to the Knight shift is negligible in our system. A last remark can be made about another effect of spin orbit coupling, known as the " $p_{1 / 2}$ corrections". ${ }^{18}$ The $s_{1 / 2}, p_{1 / 2}, p_{3 / 2}$, etc... orbitals are used to describe systems in the presence of a strong spin-orbit coupling where the total momentum $\mathrm{J}=\mathrm{L}+\mathrm{S}$ is the good quantum number. The $p_{1 / 2}$ state is formed by admixing of an $s$-type contribution to the $p$ orbitals, and thus has a finite value at the nucleus site. In this sense, the density of the electronic wave function at the nuclear site $d_{B i}$ discussed in our paper may not emanate from pure $s$-states only, but may also include the "contact" contribution of these $p_{1 / 2}$ orbitals.

\section{Anisotropic shift}

The anisotropic shift measured in our work essentially comes from the dipolar interaction between the magnetic moments of the ${ }^{209} \mathrm{Bi}$ nuclei and the conduction electrons in the $p$ orbitals. We note that the diamagnetic shift coming from the orbital motion of the free electrons in a magnetic field is likely to be negligible, since the ratio between this term and the "contact" term can be approximated in simple cases by $\frac{1}{3} \frac{\left(m_{e} / m^{*}\right)}{d_{\mathrm{Bi}} \Omega} \sim 2 \times 10^{-4} \cdot{ }^{19}$

The negative sign of the anisotropic term (see Fig.3 in the main text), consistent with recent results ${ }^{10}$, suggests that a significant fraction of the $p$ orbitals (more than $\frac{2}{3}$ ) is of $p_{x y}$ rather than theoretically expected $p_{z}$ nature. Recent results of Ref. 20 reveal the presence of a non-negligible $p_{x y}$ component $(20 \%$ at the CBM and $40 \%$ at the VBM) which could be at the origin of our observation, even though this percentage is still too small to account for a sign change in the dipolar term. We additionally note that recent experiments performed on the $\mathrm{Bi}_{2} \mathrm{Se}_{3}$ surface states have also revealed a non-negligible "in-plane" component of the $p$ orbitals. ${ }^{21}$

As can be seen in figure 3 in the main text, the magnetic field behaviour of the anisotropic shift turns out to be more complex than the one of the isotropic component. If only emanating from the dipolar coupling, the anisotropic shift should also be connected to the spin polarization (see Eq. 11) and thus saturate above 16 T. An approximately linear increase with almost zero intercept is indeed observed in the anisotropic shift up to 15 T. However, the anisotropic shift keeps on increasing above this field (leading to the drop clearly observed in the total shift above $15 \mathrm{~T}$ in figure 1 of the main text) before reaching an apparent saturation at around $22 \mathrm{~T}$. At present we are not able to give a satisfying explanation for this behavior. One possibility is that, unlike the isotropic term, the dipolar term exhibits a magnetic field dependence even when the spin polarization is frozen because of magnetic-field-induced modifications of the shape of the wave function. This would however a priori not explain the saturating trend above $B=22$ T. Surprisingly, the field dependence of the anisotropic shift can be very well reproduced using the equation $\Delta f_{\text {axial }}=2 \mathcal{A}_{\text {axial }} n_{e} \times P$, where $P$ is calculated in our simple 3 DEG model (see II.A) with a $g$-factor $g_{e f f}^{*}=23$, which is the value characterizing the $g$-factor in the $(a, b)$ plane. A possible explanation for this "delayed" saturation, could be the existence of a field dependence in the spin polarization of the lowest Landau levels describing the $p$-wave states. This is precisely what is expected in the general 3D Dirac Hamiltonian for $\mathrm{Bi}_{2} \mathrm{Se}_{3}$ where the $p$-wave orbitals are composed of a mixing of Bi and Se $p$-orbitals (see Ref. 8 for more details). In this case, the second lowest Landau level tends to depolarize with increasing magnetic field, which reduces the total spin polarization and delay the expected point (magnetic field) for complete spin polarization. A deeper examination of this high field behavior would require supplementary angular dependent experiments and is out of the scope of the present paper. The complex behavior of this anisotropic component further illustrates the importance of being able to rely on the isotropic component to study the electronic spin polarization.

\section{C. ${ }^{77}$ Se shift at low magnetic field}

The ${ }^{77}$ Se NMR, which is free from quadropular coupling effects due to the $\frac{1}{2}$ nuclear spin, should $a$ priori have been more suitable to investigate the shift of the main resonance in $\mathrm{Bi}_{2} \mathrm{Se}_{3}$. However, a series of ${ }^{77} \mathrm{Se} \mathrm{NMR}$ measurements has shown that: i) the relaxation times at low temperature are very long (e.g. $\sim 0.5$ day at $60 \mathrm{~K}$ ), which makes it more complicated and time-consuming to study; ii) The Knight shift is not as strong as it is for Bi, which was therefore better adapted to probe the electronic properties of $\mathrm{Bi}_{2} \mathrm{Se}_{3}$.

The low field Knight shift at $1.5 \mathrm{~K}$ was nevertheless studied, either by single shot, or using a small tipping angle pulse. In spite of two inequivalent Se sites, we have observed only one Se signal. Figure S5 shows the spectra and shifts of the ${ }^{77} \mathrm{Se} \mathrm{NMR}$ up to $9 \mathrm{~T}$, and at $1.5 \mathrm{~K}$ with $B \| c$-axis. The shift has been calculated with respect to the bare Se gyromagnetic ratio, ${ }^{77} \gamma=8.13 \mathrm{MHz} / \mathrm{T} .{ }^{27}$

The low field frequency shift increases linearly, similarly to the Bi shift, also pointing toward a connection to the electronic spin polarization. The sign of the shift is however negative, suggesting a "dipolar" rather than "contact" origin, which is consistent with the expected absence of s-like wave function at the Se site. ${ }^{20}$ This is also responsible 


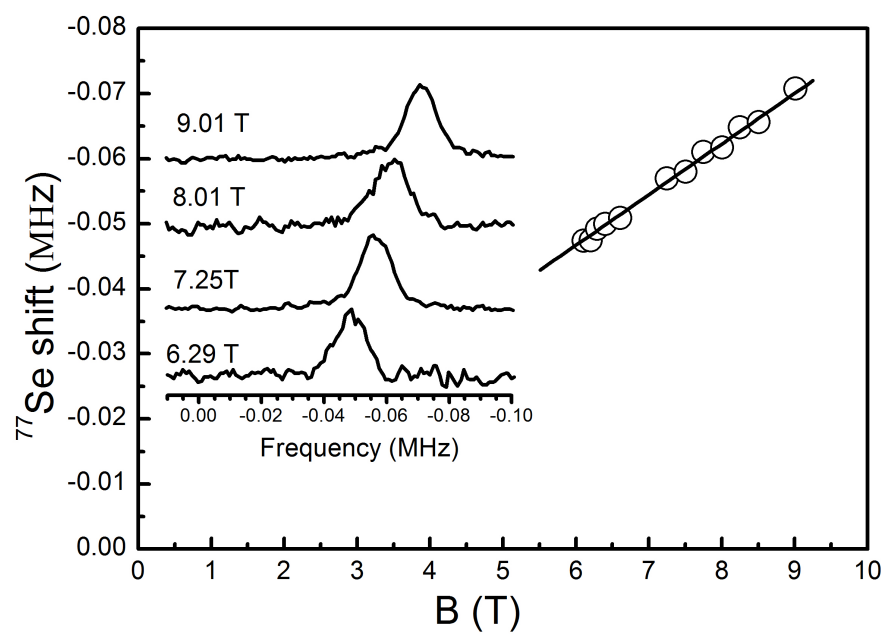

Figure S 5: Field dependence of ${ }^{77}$ Se shift at $1.5 \mathrm{~K}$ ( $B \| c$-axis). The inset shows the representative raw spectra at different magnetic fields.

for the very long relaxation time observed. Further experiments, including angular dependant NMR experiments, would be required to fully characterize the ${ }^{77} \mathrm{Se} \mathrm{NMR}$ in $\mathrm{Bi}_{2} \mathrm{Se}_{3}$. In particular, this could help our understanding of the behavior of the dipolar coupling in high magnetic fields.

\section{D. density dependence of ${ }^{209} \mathrm{Bi}$ NMR shift}

We report in this section our analysis of the density dependence of the NMR shift at low magnetic fields. In figure S6,

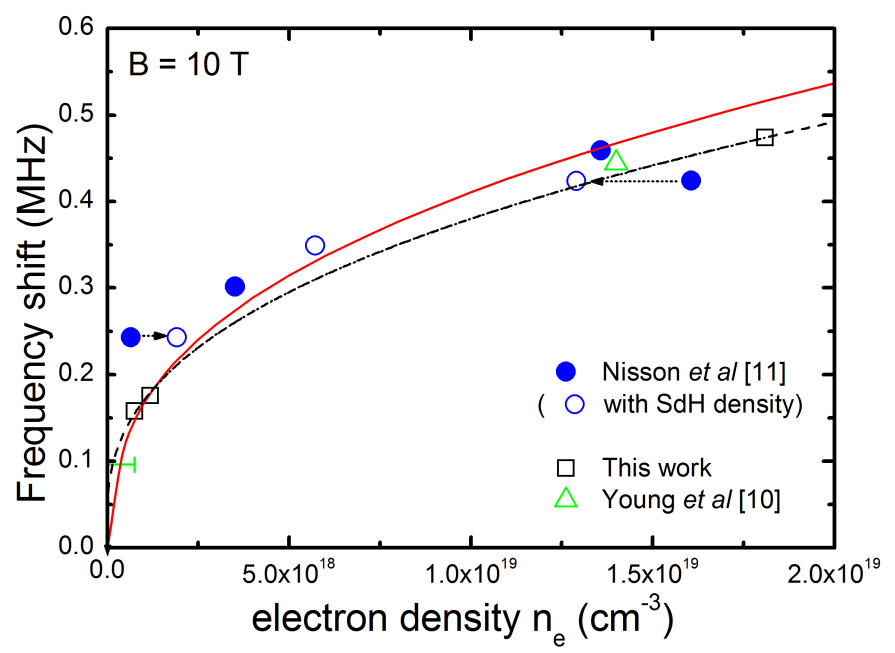

Figure S 6: Density dependence of the ${ }^{209} \mathrm{Bi}$ central line shift for our samples, together with results obtained in other recent works. The horizontal dotted arrows show where the original data points are located when the electron density is estimated from SdH oscillations (see text). The solid line is the simulated variation of shift in the 3DEG model discussed in II.A. The dashed line is a fit to our data (black squares) allowing an extra non-zero intercept.

we plot the total frequency shift at a magnetic field of $10 \mathrm{~T}$ as a function of the electron density. This magnetic field is low enough for all samples to be far from the "saturating regime" observed at high magnetic fields. We also include in this plot data recently published in Ref. 10,11. The shift is scaled to its expected value at $B=10 \mathrm{~T}$ assuming a linear field dependence in this region, which is correct for electron densities higher than the one of our sample P3-6 (for which the non-linearities of the shift due to the proximity of the "saturating regime" will only appear at higher magnetic fields). For the data of Ref. 10, the electron density was estimated using the bulk Fermi energy determined from the 
ARPES measurement reported therein. For their high density sample, we estimate $E_{F} \sim 118$ meV, which using Eq. 2 gives $n_{e} \sim 1.4 \times 10^{19} \mathrm{~cm}^{-3}$. For their low density sample, the bulk conduction band is barely visible in the ARPES scan, which points toward a very small electron density. We report the value of the shift $(\sim 96 \mathrm{kHz})$ as an horizontal bar of absciss coordinates $\left(n_{e}<7.47 \times 10^{17}\right)$ which corresponds to $E_{F}<18.5 \mathrm{meV}$, a very large upper bound for the Fermi energy in this sample. For the data of Ref. 11, the electron concentration was re-calculated directly from the oscillation frequency (as explained in I.B.) when available in the table 1 of Ref. 11. This generally gives a more reliable estimation than the one obtained from the Hall resistance which depends on various geometrical factors. The new data points (shown as open circles in Fig. S6) fall on a common trend obeyed by all samples, showing a positive correlation of the total NMR shift with the electron density. The increase of the shift with the carrier concentration is close to the previously reported $n_{e}^{1 / 3}$ behavior for $n$ and $p$-type PbSe and PbTe. ${ }^{22,23}$ In a parabolic band approximation and at low magnetic fields, the polarization of a $3 \mathrm{D}$ electron gas varies as $n_{e}^{-2 / 3}$, which multiplied by the carrier concentration gives the $n_{e}^{1 / 3}$ dependence. Our simulation, plotted as a red solid line, shows the exact quantitative result obtained when the anisotropy of the conduction is taken into account. In this simulation (detailed in II.A), the total NMR shift is directly connected to the spin polarization with a single fitting prefactor $\mathcal{A}_{\text {hyp }}$ by writing $\Delta f=\mathcal{A}_{\text {hyp }} \times n_{e} \times P$. $\mathcal{A}_{\text {hyp }}$ is an effective hyperfine coupling constant taking into account the two main contributions ("contact" and "dipolar") to the hyperfine coupling. The spin polarization has been calculated with $g_{\text {eff }}^{*}=32.8$ obtained for sample P3-6 by the field dependence of the NMR shift (see main text). Given the fact that each sample may present a different amount of disorder and a possible density-induced variation of the $g$-factor, this model catches the experimental data surprisingly well. This agreement not only shows that the NMR shift is essentially coming from the coupling to the conduction electrons, but also suggest that their degree of polarization is directly present in the total shift, as expected from the nature of the hyperfine coupling (see Eq. 11). The extracted value of the parameter $\mathcal{A}_{\text {hyp }}$ which best describes all the existing data is $\mathcal{A}_{\text {hyp }}=2.76 \pm 0.25 \times 10^{-13} \mathrm{~cm}^{3} / \mathrm{s}$, where the error bar is estimated from the scattering of the experimental data around the simulated curve. For a more complete modelling of the total shift, we have also tried to fit our experimental data allowing an extra non-zero intercept in addition to our calculated hyperfine shift, which represents the effect of a possible additional "chemical shift", independent of the conduction electrons. The fit of our data in these conditions (dashed line) yields $\mathcal{A}_{\text {hyp }}=2.34 \times 10^{-13} \mathrm{~cm}^{3} / \mathrm{s}$ and an intercept (residual shift) of $33 \mathrm{kHz}$, which is still an order of magnitude below the isotropic hyperfine component of main interest in this work. This very small intercept in the zero-density limit rules out the presence of isolated magnetic moments in the studied samples, as well as a significant direct contribution of filled electronic shells (chemical shift).

We finally note that the simplicity of this observed dependence on density, which can be very useful to characterize the total $\mathrm{NMR}$ shift in $\mathrm{Bi}_{2} \mathrm{Se}_{3}$, can to some extent be surprising. Indeed, according to the first term of Eq. 11 taken at $\theta=0^{\circ}$, the density dependence of the total frequency shift is given by the factor $n_{e} \times P$ provided $\left(\mathcal{A}_{\text {iso }}+2 \mathcal{A}_{\text {axial }}\right)$ is independent of the electron density. On the other hand, the density dependence of the isotropic and anisotropic shifts, taken separately, differs from the $n_{e}^{1 / 3}$-like behaviour expected if each coupling constant $\mathcal{A}_{\text {iso }}$ and $\mathcal{A}_{\text {axial }}$ was independent of the density. As a simple example, the anisotropic shift measured for P1-3 (high carrier density) is much smaller than its value at lower electron density, which implies that $\mathcal{A}_{\text {axial }}$ is not constant but decreases with $n_{e}$. More generally, the ratio between the isotropic and anisotropic component of the Knight shift increases with the Fermi energy (or carrier concentration), in qualitative agreement with Ref. 10. This points toward a change in the nature of the wave function as we go to higher energies in the conduction band, characterized by an increase of the $s$ character. Our observations in figure S6 suggest that the density dependence of $\mathcal{A}_{\text {axial }}$ and $\mathcal{A}_{\text {iso }}$ are apparently compensated, such that $\mathcal{A}_{\text {iso }}+2 \mathcal{A}_{\text {axial }}$ has a weak density dependence. Whether this is a simple coincidence or an effect rooted to some deeper physical arguments is an open question which is left for future works.

\section{E. Temperature dependence of ${ }^{209} \mathrm{Bi}$ central line shift}

We have performed temperature dependent NMR measurements on different samples (P2-6B,P3-6,P1-A1) for magnetic fields up to $30 \mathrm{~T}$. In figure $\mathrm{S} 7$, we report the variation of the NMR central line frequency shift as a function of temperature up to room temperature at magnetic fields of $B=9 \mathrm{~T}$ and $B=15 \mathrm{~T}$ for sample P2-6B, and P1-A1, respectively. In both cases, these magnetic fields are well below the magnetic field $B_{s}$ corresponding to the full electronic spin polarization. The P2-6B shift (left-scale) is constant a low temperature (2 K-30 K), and then smoothly drops until $T \sim 200 \mathrm{~K}$ where it tends to saturate. Sample P1-3 (right scale) shows a similar but less pronounced trend.

We note that a shift emerging from localized magnetic moments should exhibit a characteristic $1 / T$ Curie temperature dependence (shown as a dashed-line guide to the eyes in figure S7) which is clearly not observed in any of our samples at low temperature. On the other hand, the simulation of the temperature dependence of the Knight shift obtained with our 3DEG model (dotted-line, see section II.C. for the calculation details) gives a good qualitative 


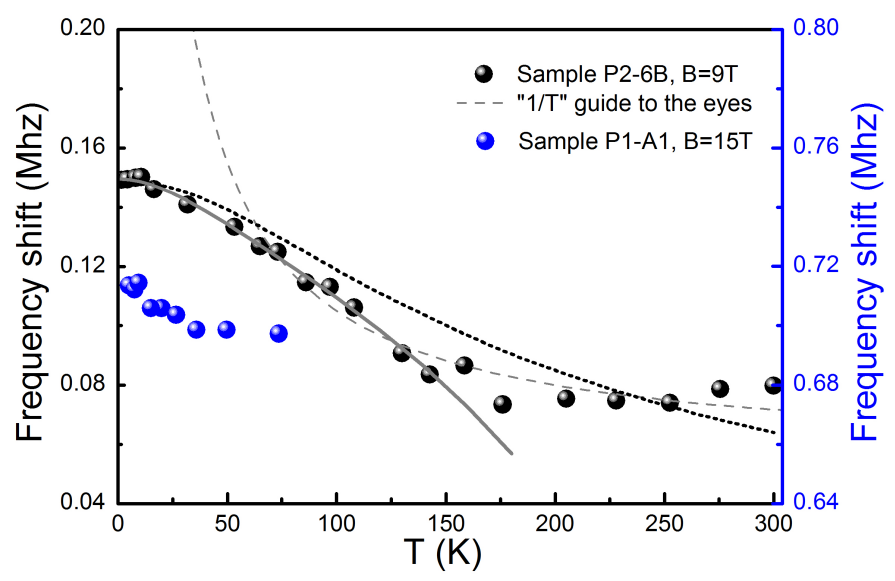

Figure S 7: Temperature dependence of the ${ }^{209}$ Bi frequency shift for samples P2-6B and P1-A1 at $B=9$ and 15 T, respectively. The dotted-line is the simulated variation of the shift using our 3DEG model (see text). The solid line further includes the effect of disorder. The dashed-line is a guide to the eyes for a $1 / T$ behavior.

description of the shift evolution for temperatures up to $\sim 100 \mathrm{~K}$. This shows that the temperature dependence of the total frequency shift can be attributed to the temperature dependence of the spin polarization, essentially coming from the smearing of the Fermi-Dirac function at the Fermi level. Including disorder (solid line), and in particular its increase with temperature inferred from the zero-field resistance, gives a very good description of the data, at least up to $\sim 150 \mathrm{~K}$. We note that a possible spin-orbit induced contribution to the shift ${ }^{17}$ should lead to a different temperature dependence ${ }^{24}$ which is not observed in our experiment. It is also not possible to fit our shift correctly with a temperature-independent term, which rules out the presence of a significant "spin-independent" orbital coupling, at least at these magnetic fields.

At higher temperature, the shift stops dropping and even shows a slight increase above $200 \mathrm{~K}$. This behavior can be attributed to the carrier activation through the $\mathrm{Bi}_{2} \mathrm{Se}_{3}$ energy gap $(0.215 \pm 5 \mathrm{meV}$, optically measured on the same samples), which can independently be observed with transport (Hall) measurements at high temperatures. This slight increase of the shift is therefore another manifestation of the dependence of the shift on the total carrier density. The smoother variation of the shift observed for sample P1-A1 is expected, since the higher Fermi energy in this sample makes the polarization more robust with respect to temperature effects. The high magnetic field temperaturedependence of the total shift has also been measured and shows a more complex behavior due to the combination of the different behavior of the isotropic and axial components in high fields.

1 I. Lifshitz and A. Kosevich, Zh. Eksp. Teor . Fiz. 29, 730 (1955), [Sov. Phys. JETP 2, 636 (1956)].

${ }^{2}$ H. Köhler, Phys. stat.sol. (b) 58, 91 (1973).

3 V. A. Kulbachinskii, N. Miura, H. Nakagawa, H. Arimoto, T. Ikaida, P. Lostak, and C. Drasar, Phys. Rev. B 59, 15733 (1999), URL http://link.aps.org/doi/10.1103/PhysRevB.59.15733.

4 K. Eto, Z. Ren, A. A. Taskin, K. Segawa, and Y. Ando, Phys. Rev. B 81, 195309 (2010), URL http://link.aps.org/doi/ 10.1103/PhysRevB.81.195309.

5 E. W. H. Köhler, Phys. stat.sol. (b) 67, 675 (1975).

6 D. Shoenberg, Magnetic oscillations in metals, p. 425 (Cambridge University Press, 1984).

7 A. Wolos, A. Drabinska, S. Szyszko, M. Kaminska, S. G. Strzelecka, A. Hruban, A. Materna, and M. Piersa, AIP Conference Proceedings 1566, 197 (2013), URL http://scitation.aip.org/content/aip/proceeding/aipcp/10.1063/1.4848353.

8 M. Orlita et al., "Magneto-optics of massive Dirac fermions in bulk $\mathrm{Bi}_{2} \mathrm{Se}_{3}$ ", in preparation.

9 W. G. Clark, M. E. Hanson, F. Lefloch, and P. Sgransan, Review of Scientific Instruments 66, 2453 (1995), URL http: //scitation.aip.org/content/aip/journal/rsi/66/3/10.1063/1.1145643.

10 B.-L. Young, Z.-Y. Lai, Z. Xu, A. Yang, G. D. Gu, Z.-H. Pan, T. Valla, G. J. Shu, R. Sankar, and F. C. Chou, Phys. Rev. B 86, 075137 (2012), URL http://link.aps.org/doi/10.1103/PhysRevB.86.075137.

11 D. M. Nisson, A. P. Dioguardi, P. Klavins, C. H. Lin, K. Shirer, A. C. Shockley, J. Crocker, and N. J. Curro, Phys. Rev. B 87, 195202 (2013), URL http://link.aps.org/doi/10.1103/PhysRevB.87.195202.

12 A. Willig and B. Sapoval, Solid State Communications 11, 1077 (1972), ISSN 0038-1098, URL http://www.sciencedirect. com/science/article/pii/0038109872903249.

13 G. A. Miranda, J. A. McNeil, and W. G. Clark, Phys. Rev. B 9, 495 (1974), URL http://link.aps.org/doi/10.1103/ 
PhysRevB.9.495.

14 D. P. Tunstall, Journal of Physics C: Solid State Physics 21, 2853 (1988), URL http://stacks.iop.org/0022-3719/21/i= $15 / a=017$.

15 B. Sapoval and J. Y. Leloup, Phys. Rev. B 7, 5272 (1973), URL http://link.aps.org/doi/10.1103/PhysRevB.7.5272.

16 G. Carter, L. Bennett, and D. Kahan, Prog. Mater. Sci. 20, 11 (1977).

17 G. S. Tripathi, L. K. Das, P. K. Misra, and S. D. Mahanti, Phys. Rev. B 25, 3091 (1982), URL http://link.aps.org/doi/ 10.1103/PhysRevB.25.3091.

18 P. Larson, Phys. Rev. B 68, 155121 (2003), URL http://link.aps.org/doi/10.1103/PhysRevB.68.155121.

19 J. E. Hebborn, Proceedings of the Physical Society 80, 1237 (1962), ISSN 0370-1328, URL http://dx.doi.org/10.1088/ 0370-1328/80/6/304.

20 A. Pertsova and C. M. Canali, arXiv:1311.0691v1 (2013).

21 Y. Cao, J. A. Waugh, X.-W. Zhang, J.-W. Luo, Q. Wang, T. J. Reber, S. K. Mo, Z. Xu, A. Yang, J. Schneeloch, et al., Nat. Phys. 9, 499 (2013), ISSN 1745-2473.

22 J. Y. Leloup, B. Sapoval, and G. Martinez, Phys. Rev. B 7, 5276 (1973), URL http://link.aps.org/doi/10.1103/ PhysRevB.7.5276.

23 B. Sapoval, J. Phys. Colloques 29, C4 (1968), URL http://dx.doi.org/10.1051/jphyscol: 1968419.

24 S. Misra, G. S. Tripathi, and P. K. Misra, Journal of Physics C: Solid State Physics 20, 277 (1987).

${ }^{25}$ H. Duddeck, Progress in Nuclear Magnetic Resonance Spectroscopy 27, 1 (1995), ISSN 0079-6565, URL http://www . sciencedirect.com/science/article/pii/007965659400005F.

${ }^{26}$ R. E. Taylor, B. Leung, M. P. Lake, and L.-S. Bouchard, The Journal of Physical Chemistry C 116, 17300 (2012), http://dx.doi.org/10.1021/jp307051z.

27 We note that the ${ }^{77}$ Se gyromagnetic ratio is not unambiguously determined in the literature (see e.g. Ref. 25), which can cause a significant difference in the magnitude and even the sign of the resulting shifts. This is at the origin of the large difference between the shift reported in figure S5 and the one obtained in Ref. 26. The conclusion drawn from the present value of the ${ }^{77}$ Se shift is therefore based on the assumption that the averaged value of $8.13 \mathrm{MHz} / \mathrm{T}$ is relevant in our system. 